\title{
A non-integer order dengue internal transmission model
}

\author{
Zain UI Abadin Zafar ${ }^{1,2^{*}}$, Muhammad Mushtaq ${ }^{1}$ and Kashif Rehan ${ }^{3}$
}

\author{
"Correspondence: \\ zainzafar2016@hotmail.com; \\ zainzafar@ucp.edu.pk \\ ${ }^{1}$ Department of Mathematics, \\ University of Engineering and \\ Technology, Lahore, Pakistan \\ ${ }^{2}$ Faculty of Information Technology, \\ University of Central Punjab, Lahore, \\ Pakistan \\ Full list of author information is \\ available at the end of the article
}

\begin{abstract}
A non-linear mathematical model with non-integer order $\gamma, 0<\gamma \leq 1$, is used to analyze the dengue virus transmission in the human body. Both disease-free $\mathcal{F}_{0}$ and endemic $\mathcal{F}^{*}$ equilibria are calculated. Their stability is also described using the stability theorem of non-integer order. The threshold parameter $\mathcal{R}_{0}$ demonstrates an important behavior in the stability of a considerable model. For $\mathcal{R}_{0}<1$, the disease-free equilibrium (DFE) $\mathcal{F}_{0}$ is an attractor. For $\mathcal{R}_{0}>1, \mathcal{F}_{0}$ is not stable, the endemic equilibrium (EE) $\mathcal{F}^{*}$ exists, and it is an attractor. Numerical examples of the proposed model are also proven to study the behavior of the system.
\end{abstract}

Keywords: dengue model; fractional derivatives; stability; predictor-corrector method; Grunwald-Letnikov method

\section{Introduction}

Dengue viral diseases are a standout amongst the supreme critical mosquito-borne maladies these days. They create problems like dengue fever (DF), dengue hemorrhagic fever (DHF), and dengue stun disorder (DSS) or dengue hemorrhagic fever (DHF). Lately, the frequency of DHF has expanded significantly. Dengue may be caused by one of the serotypes DEN-1 to DEN-4. For the most part, septicity with one serotype presents upcoming defensive resistance against that specific serotype yet not against different serotypes. When anyone is infected for the second time with various serotypes, a serious disease will occur [1]. After an infected mosquito bites, the virus enters the human body and repeats inside the cell of the mononuclear phagocyte ancestry (monocytes, macrophages, and B cell). The incubation time frame is 7-10 days. Then a viremia stage, where the patient is plainly febrile and infective, takes place. From that point, the infected human body may either recuperate or advance to the leakage stage, prompting DHF and/or potentially DSS [2]. To calculate the span of viremia, analysts' assumed that noticeable viremia began on the eve of onset of ailment, and after the recognition of the disease in the human body, it vanishes soon.

Non-integer calculus characterizes a speculation of conventional integration, differentiation to the fractional number and complex order. For instance, control theory, viscoelasticity, electricity, heat conduction, fractals, chaos, etc. searching real frameworks examples portray by the non-integer derivative is an open problem in the field of non-integer calculus [3]. The generalization of differential calculus to the fractional order of derivatives can be followed back to Leibnitz. It can help us to decrease inaccuracies emerging

(c) The Author(s) 2018. This article is distributed under the terms of the Creative Commons Attribution 4.0 International License (http://creativecommons.org/licenses/by/4.0/), which permits unrestricted use, distribution, and reproduction in any medium, provided you give appropriate credit to the original author(s) and the source, provide a link to the Creative Commons license, and indicate if changes were made. 
from the ignored parameters in the modeling of real-life problems. Different applications, like in the anomalous electron transport in amorphous materials, the reaction kinetics of proteins, the irregular electron transference in undefined materials, the di-electrical or mechanical relation of polymers, the demonstrating of glass framing fluids, and in many other fields, are effectively achieved in various articles [4]. The principle purpose behind utilizing non-fractional order models was the non-appearance of arrangement techniques for non-integer DEs. It is an evolving field in the area of mathematical physics and applied mathematics, like chemistry, biology, economics, and image and signal dispensation. It has several uses in numerous fields of engineering and science. The calculus of variations is broadly used for some disciplines such as pure mathematics, engineering, and applied mathematics. In addition, the analysts have lately revealed that the physical frameworks with dissipation can be unmistakably modeled more precisely by utilizing non-integer representations [5].

Derivatives of fractional order involve more information about the system in study than integer order derivatives, which are local operators. The physical and geometrical importance of the fractional integral containing the complex and real conjugate power-law exponent has been proposed. One physical connotation of the non-integer order in non-integer derivatives is that of the file (index) of memory [6]. The memory property is very useful in modeling of several phenomena. The state of infection at a given moment $t$ depends on the state before $t$, namely $t-1, t-2, \ldots$. In this sense fractional calculus may help to distinguish distinct routes in (dengue) infection in different patients. We note that, for smaller value of $\gamma$, the variable approaches the corresponding asymptotic values faster [7].

Additionally, non-integer calculus shows a vital part of superdiffusive and subdiffusive procedures, which make it a helpful instrument in the study of disease transmission [8]. Since integer order differential conditions cannot decisively portray the exploratory and field estimation information, alternative tactic fractional order differential equation models are presently being widely applied $[9,10]$. The upside of non-integer order differential equation systems over ordinary differential equation frameworks is that they allow more noteworthy degrees of flexibility and incorporate memory effect in the model. In other words, they give a magnificent device for the portrayal of memory and traditional chattels which were not considered in the established non-fractional order prototypes [11]. Fractional calculus has heretofore been applied in epidemiological investigations [12-15]. Also, fractional order models possess memory, fractional order differential equations give us a more realistic way to model the dengue system. Lately, they have been utilized to analyze a dengue plague prototype [16]. Regardless of the way that the operator of the non-integer is more complex than the traditional one, there occur numerical strategies for cracking systems of DEs which are nonlinear [17].

Purohit and Kalla [18] discussed the generalized non-integer PDEs containing the Liouville space non-integer derivatives and the Caputo time non-integer derivatives. The elucidations of these equations were attained using the Laplace and Fourier transforms. Also, Purohit [19] discussed the generalized non-integer partial differential including the Hilfer time non-integer derivative and the space non-integer generalized Laplace operators occurring in quantum mechanics. Chouhan et al. [20] presented the technique for developing the result of the generalized forms of non-integer DE and Volterra-type DE. Nisar et al. [21] discussed a generalized non-integer kinetic equation containing a gener- 
alized Bessel function of the first kind. Also, some of the interesting nonlinear models and fractional models were discussed in [22-35].

Lately, the majority of the dynamical frameworks based on the non-fractional order calculus have been changed into the non-integer order domain. Because of the additional degrees of opportunity and the adaptability which can be utilized to decisively fit the test information much more superior to anything in the integer order modeling, the concept of fractional calculus has become an alternative mathematical method to describe models with non-local behavior. These models represented by fractional differential equations contain the historical memory and global information of physical problems. In fact, the fractional approach generalizes the classical models of the dengue model. The purpose of this modification is to have better understanding and prediction of epidemic patterns and intervention measures.

The fractional order models are said to be useful in distinguishing distinct patterns in patients' disease progression and possibly provide better fit data. Clinicians may use the information from the general fractional order system to devise new treatments to each individual in particular by fitting his/her data with the most appropriate fixed index. We analyze the model's behavior for distinct values of the order of the fractional derivative $\gamma$ and for biologically relevant parameters, namely the ones related with the infection and treatment [7]. Also biological systems have fractal structures and very close ties with fractional equations. Thus using the fractional differential equation for this system can produce natural results. A more reliable model can be obtained by choosing a relevant fractional index according to available real data.

This article is organized into five sections. The introduction is the first section in which we elaborate on some history of fractional calculus. In Section 2, we give notations related to the concept of FDEs. In Section 3, we ponder on the fractional order model linked with the dynamics of dengue model. Qualitative dynamics of the considerable system is resolute using an elementary reproduction number. We provide a comprehensive investigation of the global asymptotical stability of the DFE point and the native asymptotical stability of the EE point. In Section 4, numerical imitations are offered to validate the main outcomes, and conclusion is drawn in Section 5.

\section{Inceptions}

For several ages, there have been numerous demarcations that apt the notion of fractional derivatives [36, 37]. In this article, the Caputo (C), Riemann-Liouville (RL), and GrunwaldLetnikov (GL) fractional derivative (FD) demarcations are presented. Firstly, we present the demarcation of the RL non-integer integral

$$
J^{\varsigma} g(z)=(\Gamma(\varsigma))^{-1} \int_{0}^{z} \frac{g(s)}{(z-s)^{1-\gamma}} d s
$$

where $\gamma>0, g \in L^{1}\left(R^{+}\right)$, and $\Gamma(\cdot)$ is the gamma function.

The $R L$ derivative is given by

$$
D_{R}^{\gamma} g(z)=\frac{d^{m}}{d z^{m}}\left[J^{m-\gamma} g(z)\right]=\frac{1}{\Gamma(m-\gamma)} \frac{d^{m}}{d z^{m}} \int_{0}^{z} \frac{g(s)}{(z-s)^{1-m+\gamma}} d s, \quad m-1 \leq \gamma<m .
$$


The Caputo fractional derivative is agreed to be as follows:

$$
D_{C}^{\gamma} g(x)=J^{M-\varsigma}\left[\frac{d^{M}}{d x^{M}} g(x)\right]=\frac{1}{\Gamma(M-\varsigma)} \int_{0}^{x}(x-s)^{M-\varsigma-1} g^{(M)}(s) d s,
$$

where $M>\gamma, \forall M \in Z^{+}$.

The GL derivative is agreed to be

$$
{ }_{a} D_{x_{k}}^{\gamma} g(x)=\lim _{h \rightarrow 0} \frac{1}{h^{\gamma}} \sum_{j=0}^{\left[\frac{x-a}{h}\right]}(-1)^{j}\left(\begin{array}{l}
\gamma \\
j
\end{array}\right) g(x-j h),
$$

where [.] means the integer part.

The Laplace transform of the Caputo FD is specified by

$$
\mathcal{L}\left[D_{C}^{\varphi} g(x)\right]=s^{\varphi} G(s)-\sum_{j=0}^{n-1} g^{(j)}(0) s^{\varphi-j-1} .
$$

The Mittag-Leffler function (MLF) is considered by using infinite power series:

$$
E_{\alpha, \beta}(s)=\sum_{k=0}^{\infty} \frac{s^{k}}{(\alpha k+\beta)} .
$$

The Laplace transform of the functions is

$$
\mathcal{L}\left[t^{\beta-1} E_{\alpha, \beta}\left( \pm a t^{\alpha}\right)\right]=\frac{s^{\alpha-\beta}}{s^{\alpha} \mp a} .
$$

Let $\alpha, \beta>0$ and $z \in \mathbb{C}$, and the Mittag-Leffler functions satisfy the equality given by Theorem 4.2 in [36]:

$$
E_{\alpha, \beta}(z)=z E_{\alpha, \alpha+\beta}(z)+\frac{1}{\Gamma(\beta)} .
$$

Definition 1 ([22]) "A function $F$ is Holder continuous if there are non-negative amounts $W, v$ such that

$$
\|F(s)-F(t)\| \leq W\|s-t\|^{v}
$$

for all $s, t$ in the purview of $F$ and $v$ is the Holder exponent. We represent the space of Holder-continuous functions by $W^{0, v}$.

We improve a generalized inequality, in which the core appraisal system is a vector fractional order system.

A non-negative vector $v$ means that each constituent of $v$ is non-negative. We represent a non-negative vector by $0 \leq \leq \nu$.

Consider the fractional order system:

$$
D_{C}^{\varphi} \varpi(\mathfrak{H})=z(\mathfrak{H}, \varpi),
$$


with the initial condition $\varpi(0)=\varpi_{0}$, where $D_{C}^{\varsigma} \varpi(\mathfrak{H})=\left(D_{C}^{\varsigma} \varpi_{1}(\mathfrak{H}), D_{C}^{\varsigma} \varpi_{2}(\mathfrak{H}), D_{C}^{\varsigma} \varpi_{3}(\mathfrak{H}), \ldots\right.$, $\left.D_{C}^{\varsigma} \varpi_{m}(\mathfrak{H})\right)^{T}, 0<\varsigma<1, \varpi(H) \in \mathcal{F} \subset R^{m}, \mathfrak{H} \in[0, T)(T \leq \infty), \mathcal{F}$ is an open set, $0 \in \mathcal{F}$, and $z:[0, T) \times \mathcal{F} \rightarrow R^{m}$ is continuous in $\mathfrak{H}$ and mollifies the Lipschitz condition

$$
\left\|z\left(\mathfrak{H}, \varpi^{\prime}\right)-z\left(\mathfrak{H}, \varpi^{\prime \prime}\right)\right\| \leq P\left\|\varpi^{\prime}-\varpi^{\prime \prime}\right\|, \quad \mathfrak{H} \in[0, T)
$$

for all $\varpi^{\prime}, \varpi^{\prime \prime} \in \Omega \subset \mathcal{F}$, where $P>0$ is a Lipschitz constant.

Theorem $1([3,22])$ "Let the solution of $(10)$ be $u(\mathfrak{H}), \mathfrak{H} \in[0, H)$. If there exists a vector function $\varpi=\left(\varpi_{1}, \varpi_{2}, \ldots, \varpi_{m}\right)^{H}:[0, H) \rightarrow \mathcal{F}$ such that $\varpi_{i} \in G^{0, v}, \varsigma<\varpi<1, i=1,2, \ldots, m$, and

$$
D_{C}^{\varphi} \varpi \leq \leq g(\mathfrak{H}, \varpi), \quad \mathfrak{H} \in[0, H]
$$

If $\varpi(0) \leq \leq u_{0}, u_{0} \in \mathcal{F}$, then $w \leq \leq u, \mathfrak{H} \in[0, H]$.

Let $g: \mathcal{F} \rightarrow R^{m}, \mathcal{F} \in R^{m}$, we consider the following system of fractional order:

$$
D_{C}^{\varsigma} x(t)=g(x), \quad x(0)=x_{0} .
$$

Definition 2 ([22]) "We say that $\mathcal{F}$ is an equilibrium point of (13) if and only if $g(\mathcal{F})=0$ ".

Remark 1 When $\varsigma \in(0,1)$, the fractional system $D_{C}^{\varsigma} x(t)=g(x)$ has the identical equilibrium points as the arrangement $\frac{d x(t)}{d t}=g(x)$.

Definition 3 ([22]) "The equilibrium point $\mathcal{F}$ of autonomous (13) is said to be stable if, for all $\epsilon>0, \varepsilon>0$ exists such that if $\left\|x_{0}-\mathcal{F}\right\|<\varepsilon$, then $\|x-\mathcal{F}\|<\epsilon, t \geq 0$; the equilibrium point $\mathcal{F}$ of autonomous (13) is said to be asymptotically unwavering if $\lim _{t \rightarrow \infty} x(t)=\mathcal{F}^{\prime \prime}$.

Theorem 2 ([22, 38]) "The equilibrium points of system (13) are locally asymptotically stable if all eigenvalues $\lambda_{i}$ of the Jacobian matrix $J$, calculated in the equilibrium points, satisfy $\left|\arg \left(\lambda_{i}\right)\right|>\varsigma \frac{\pi}{2}$.

Here are a few newly developed various definitions of fractional derivative including one-parameter and two-parameter fractional derivatives:

Definition 4 ([39]) “The Caputo fractional time derivative $\left(\mathrm{UFD}_{t}\right)$ of order $\alpha$ is given by

$$
\mathfrak{D}_{t}^{(\alpha)}(f(t))=\frac{1}{\Gamma(1-\alpha)} \int_{0}^{t}(t-\tau)^{-\alpha} \dot{f}(\tau) d \tau
$$

with $\alpha \in[0,1]$ and $a \in[-\infty, t), f \in H^{1}(a, b), b>a$.

Definition 5 ([39]) "If we change the kernel $(t-\tau)^{-\alpha}$ with the function $e^{-\frac{\alpha}{1-\alpha} t}$ and $\frac{1}{\Gamma(1-\alpha)}$ with $\frac{M(\alpha)}{1-\alpha}$, we obtain the following new definition of fractional time derivative $\left(\mathrm{NFD}_{t}\right)$ :

$$
\mathfrak{D}_{t}^{(\alpha)}(f(t))=\frac{M(\alpha)}{(1-\alpha)} \int_{a}^{t} e^{-\frac{\alpha(t-\tau)}{1-\alpha}} \dot{f}(\tau) d \tau
$$


where $M(\alpha)$ is a normalization function such that $M(0)=M(1)=1$. According to definition (15), the $\mathrm{NFD}_{t}$ is zero when $f(t)$ is constant as, in the $\mathrm{UFD}_{t}$, the kernel does not have singularity for $t=\tau$ ".

Definition 6 ([40]) "Let $u$ be a function in $H^{1}(a ; b) ; b>a ; \alpha \in[0 ; 1]$, then the new Caputo derivative of a fractional derivative of order $\alpha$ is defined as follows:

$$
\mathrm{CF}^{(\alpha)}(u(t))=\frac{M(\alpha)}{(1-\alpha)} \int_{0}^{t} e^{-\frac{\alpha(t-\tau)}{1-\alpha}} \dot{u}(\tau) d \tau
$$

where $M(\alpha)$ is a normalization function such that $M(0)=M(1)=1$. However, for the function that does not belong to $H^{1}(a ; b)$, we defined its Caputo-Fabrizio fractional as

$$
\mathrm{CF}_{\mathfrak{D}_{t}^{(\alpha)}}(u(t))=\frac{\alpha M(\alpha)}{(1-\alpha)} \int_{0}^{t} e^{-\frac{\alpha(t-\tau)}{1-\alpha}}(u(t)-u(\tau)) d \tau
$$

The definition of Caputo-Fabrizio fractional derivative (CFFD) was improved by Losada and Nieto [41] to become

$$
{ }^{\mathrm{CF}} \mathfrak{D}_{t}^{(\alpha)}(u(t))=\frac{(2-\alpha) M(\alpha)}{2(1-\alpha)} \int_{0}^{t} e^{-\frac{\alpha(t-\tau)}{1-\alpha}} \dot{u}(\tau) d \tau
$$

where $M(\alpha)$ is a normalization constant depending on $\alpha$. The CFFD has no singular kernel due to the substitution of the kernel $(t-\tau)^{-\alpha}$ appearing in the classical definition".

Definition 7 ([42]) "A great development of fractional calculus theory was made possible by the introduction of a number of variants of the Mittag-Leffler function since the early 1900s. One of them is the two-parameter Mittag-Leffler function

$$
E_{\alpha, \beta}(z)=\sum_{k=0}^{\infty} \frac{z^{k}}{\Gamma(\alpha k+\beta)}, \quad \alpha, \beta, z \in \mathbb{C}, \mathfrak{R}(\alpha)>0, \mathfrak{R}(\beta)>0,
$$

also called the generalized Mittag-Leffler function. It has been improved by many researchers. On the top of being a non-local function, the two-parameter Mittag-Leffler function plays a very vital role in the theory of fractional calculus".

Definition 8 ([42]) "Let $u$ be a function in $H^{1}(a ; b) ; b>a ; \alpha \in[0 ; 1], \beta \in(0,+\infty)$, then the Caputo-sense two-parameter fractional derivative of order $\alpha$ knowing $\beta$ (or simply the 2-GC derivative of order $\alpha$ knowing $\beta$ ) is defined as

$$
{ }_{a}^{\mathrm{gc}} D_{t}^{\alpha, \beta} u(t)=\frac{\beta W(\alpha, \beta)}{(\beta-\alpha)} \int_{a}^{t} \dot{u}(t)(t-\tau)^{\beta-1} \times E_{\alpha, \beta}\left[-\frac{\alpha \beta(t-\tau)^{\alpha}}{\beta-\alpha}\right] d \tau,
$$

where $W(\alpha, \beta)$ is a two-variable normalization function such that $W(0,1)=W(1,1)=1$.

It is obvious to see that 2-GC derivative of any order $\alpha$ knowing $\beta$ is zero when $u(t)$ is constant and its kernel does not have any singularity at $t=\tau$. Moreover, the kernel is non-local due to the two-parameter Mittag-Leffler function $E_{\alpha, \beta}\left[-\frac{\alpha \beta(t-\tau)^{\alpha}}{\beta-\alpha}\right]$. 
If the function $u$ does not belong to $H^{1}(a ; b)$, then for any function $u \in L^{1}(-\infty, b)$, its 2-GC derivative of order $\alpha$ knowing $\beta$ is defined as

$$
{ }_{a}^{\mathrm{gc}} D_{t}^{\alpha, \beta} u(t)=\frac{\alpha W(\alpha, \beta)}{(\beta-\alpha)} \int_{a}^{t}(u(t)-u(\tau))(t-\tau)^{\beta-1} \times E_{\alpha, \beta}\left[-\frac{\alpha \beta(t-\tau)^{\alpha}}{\beta-\alpha}\right] d \tau .
$$

Note that (18) can be rewritten as

$$
{ }_{a}^{g c} D_{t}^{\alpha, \beta} u(t)=\frac{\beta W(\alpha, \beta)}{(\beta-\alpha)}(\dot{u}(t) * v(t)),
$$

where

$$
v(t)=t^{\beta-1} E_{\alpha, \beta}\left[-\frac{\alpha \beta t^{\alpha}}{\beta-\alpha}\right],
$$

and $*$ the usual Laplace convolution operator giving the convolution integral with two casual functions is expressed as

$$
u(t) * v(t)=\int_{a}^{t} u(\tau) v(t-\tau) d \tau
$$

This property is fundamental for filtering and image processing”.

Definition 9 ([42]) "Let $u$ be a function in $H^{1}(a ; b) ; b>a ; \alpha \in[0 ; 1], \beta \in(0,+\infty)$, then the Riemann-Liouville-sense two-parameter fractional derivative of order $\alpha$ knowing $\beta$ (or simply the 2-GRL derivative of order $\alpha$ knowing $\beta$ ) is defined as

$$
{ }_{a}^{\mathrm{gr}} D_{t}^{\alpha, \beta} u(t)=\frac{\beta W(\alpha, \beta)}{(\beta-\alpha)} \frac{d}{d t} \int_{a}^{t} u(t)(t-\tau)^{\beta-1} \times E_{\alpha, \beta}\left[-\frac{\alpha \beta(t-\tau)^{\alpha}}{\beta-\alpha}\right] d \tau,
$$

where $W(\alpha, \beta)$ is a two-variable normalization function such that $W(0,1)=W(1,1)=1$. Equation (22) can be written as

$$
{ }_{a}^{\mathrm{gr}} D_{t}^{\alpha, \beta} u(t)=\frac{\beta W(\alpha, \beta)}{(\beta-\alpha)} \frac{d}{d t}(u(t) * v(t))
$$

where $v(t)$ is the same as (21)".

Definition 10 ([43]) "Given a function $f:[0, \infty) \rightarrow \mathbb{R}$. Then the conformable fractional derivative of $\mathrm{f}$ of order $\alpha$ is defined by

$$
T_{\alpha}(f)(t)=\lim _{\varepsilon \rightarrow 0} \frac{f\left(t+\varepsilon t^{1-\alpha}\right)}{\varepsilon}
$$

for all $t>0, \alpha \in(0,1)$. If $f$ is $\alpha$-differentiable in some $(0, a), a>0$, and $\lim _{t \rightarrow 0^{+}} f^{\alpha}(t)$ exists, then define $f^{\alpha}(0)=\lim _{t \rightarrow 0^{+}} f^{\alpha}(t)$.

Note that $T_{\alpha}(f)(t)=f^{\alpha}(t)$ denotes the conformable fractional derivatives of $f$ of order $\alpha$. If the conformable fractional derivative of $f$ of order $\alpha$ exists, then we simply say that $f$ is $\alpha$-differentiable. Since it is given that $T_{\alpha}\left(t^{p}\right)=p t^{p-\alpha}$. Further, this definition coincides with the classical definitions of RL and of Caputo fractional derivatives on polynomials (up to constant multiple)". 
Figure 1 Flow chart.

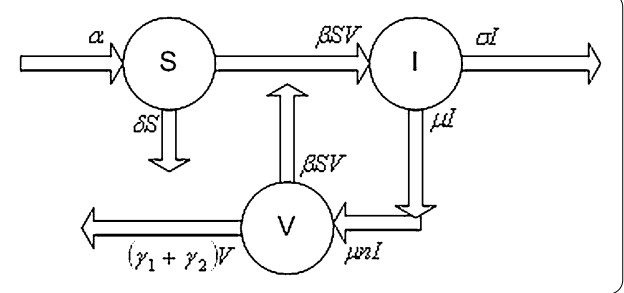

\section{Mathematical model}

To develop the equations, the dengue viruses are virulent and no other microorganism that bout human body. It is thought that macrophages, monocytes and other cells of reticuloendothelial origin primarily support dengue virus infections (in vivo) [44]. The susceptible cell is denoted by $S$, the infected cell by $I$, and the free virus by $V$. The transmission virus in the patient is described in the flow chart (Figure 1).

The model in ordinary differential equation is [45]

$$
\left\{\begin{array}{l}
\frac{d S}{d \tau}=\alpha-\beta S(\tau) V(\tau)-\delta S(\tau), \\
\frac{d I}{d \tau}=\beta S(\tau) V(\tau)-\sigma I(\tau), \\
\frac{d V}{d \tau}=\mu_{n} I(\tau)-\left(p_{1}+p_{2}\right) V(\tau)-\beta S(\tau) V(\tau) .
\end{array}\right.
$$

In this model, we used the assumption that all the parameters are positive constants. We utilized the greenest supposition that susceptible cells are formed at a constant rate $\alpha$ and expire at a rate $\delta S(\tau)$. Free virus particles infect susceptible cells at a rate corresponding to the product of their plenitudes $\beta S(\tau) V(\tau)$. The steady rate $\beta$ pronounces the viability of this process, including the rate and likelihood of successful septicity. Septic cells yield free virus at a rate proportional to their plenitude $\mu_{n} I(\tau)$, with $n$ being the multiplication rate, and free infection particles are expelled from the system at a rate $\left(p_{1}+p_{2}\right) V(\tau)$, where $\gamma_{1}$ is the natural demise rate of virus and $\gamma_{2}$ is the death rate of virus by $T$-cells. The free virus also moves to the susceptible cells compartment as $\beta S(\tau) V(\tau)$. The infected cells bite the dust at a rate $\sigma I(\tau)$. We consider this model as a model of virus dynamics depicted in [46].

\subsection{Fractional order model}

These days, a substantial attention to the fractional calculus has been shown, which allows us to consider integration and differentiation of any order. The usefulness of a FDE system is that it allows the users to handle the greater degree of freedom. The new system of FDEs to the dengue model is as follows:

$$
\left\{\begin{array}{l}
\frac{d^{\gamma_{1} S}}{d \tau^{\gamma_{1}}}=\alpha-\beta S(\tau) V(\tau)-\delta S(\tau) \\
\frac{d^{\gamma_{2} I}}{d \tau^{\gamma_{2}}}=\beta S(\tau) V(\tau)-\sigma I(\tau) \\
\frac{d^{\gamma_{3}} V}{d \tau^{\gamma_{3}}}=\mu_{n} I(\tau)-\left(p_{1}+p_{2}\right) V(\tau)-\beta S(\tau) V(\tau), \quad \gamma \in(0,1) .
\end{array}\right.
$$

The system is called commensurate if $\gamma=\gamma_{1}=\gamma_{2}=\gamma_{3}$; otherwise, it is incommensurate. Chaotic behavior of the model is noted when the total order of the system is less than three and it is connected to the fractal phase space in dynamics. If $\gamma_{1}=\gamma_{2}=\gamma_{3}=1$, the system will be that of nonlinear ordinary differential equations as presented in [45]. The region of 
stability of the non-integer order system as reviewed in $[38,47,48]$ is the region in which the system eigenvalues $\lambda$ of the characteristic equation obtained from the Jacobian matrix of system (25) at a certain equilibrium point satisfy $\left|\arg \left(\lambda_{i}\right)\right|>\gamma_{i} \pi / 2, i=1,2,3$.

\subsection{Equilibrium points and stability}

The equilibrium points of (25) are attained by cracking the nonlinear algebraic equations

$$
D^{\gamma_{1}} S(\tau)=D^{\gamma_{2}} I(\tau)=D^{\gamma_{3}} V(\tau)=0
$$

System (25) has a disease-free equilibrium point $\mathcal{F}_{0}\left(\frac{\alpha}{\delta}, 0,0\right)$ if $\mathcal{R}_{0}<1$, while if $\mathcal{R}_{0}>1$, there is, in addition to $\mathcal{F}_{0}$, a positive endemic equilibrium $\mathcal{F}^{*}\left(S^{*}, I^{*}, V^{*}\right)$ and the values of $S^{*}, I^{*}$, and $V^{*}$ are as follows:

$$
\begin{aligned}
& S^{*}=\frac{\sigma\left(p_{1}+p_{2}\right)}{\beta\left(\mu_{n}-\sigma\right)}=\frac{\alpha}{\delta \mathcal{R}_{0}}, \quad I^{*}=\frac{\alpha \beta\left(\mu_{n}-\sigma\right)-\sigma \delta\left(p_{1}+p_{2}\right)}{\sigma \beta\left(\mu_{n}-\sigma\right)}=\frac{\alpha\left(\mathcal{R}_{0}-1\right)}{\sigma \mathcal{R}_{0}}, \\
& V^{*}=\frac{\alpha \beta\left(\mu_{n}-\sigma\right)-\sigma \delta\left(p_{1}+p_{2}\right)}{\sigma \beta\left(p_{1}+p_{2}\right)}=\frac{\delta}{\beta}\left(\mathcal{R}_{0}-1\right),
\end{aligned}
$$

where $\mathcal{R}_{0}$ is the basic reproduction number defined in [45] as follows:

$$
\mathcal{R}_{0}=\frac{\alpha \beta\left(\mu_{n}-\sigma\right)}{\sigma \delta\left(p_{1}+p_{2}\right)}
$$

The value that $\mathcal{R}_{0}$ takes can signpost the situations wherein an epidemic is conceivable. Threshold quantity $\left(\mathcal{R}_{0}\right)$ is used to analyze the stability of system (25).

\section{3 $\mathcal{R}_{0}$ sensitivity analysis}

To check the sensitivity of $\mathcal{R}_{0}$ for each parameter,

$$
\begin{aligned}
& \frac{\partial \mathcal{R}_{0}}{\partial \alpha}=\frac{\beta\left(\mu_{n}-\sigma\right)}{\sigma \delta\left(p_{1}+p_{2}\right)}>0, \\
& \frac{\partial \mathcal{R}_{0}}{\partial \beta}=\frac{\alpha\left(\mu_{n}-\sigma\right)}{\sigma \delta\left(p_{1}+p_{2}\right)}>0, \\
& \frac{\partial \mathcal{R}_{0}}{\partial \mu_{n}}=\frac{\alpha \beta}{\sigma \delta\left(p_{1}+p_{2}\right)}>0, \\
& \frac{\partial \mathcal{R}_{0}}{\partial \delta}=\frac{-\alpha \beta\left(\mu_{n}-\sigma\right)}{\sigma \delta^{2}\left(p_{1}+p_{2}\right)}<0, \\
& \frac{\partial \mathcal{R}_{0}}{\partial p_{1}}=\frac{-\alpha \beta\left(\mu_{n}-\sigma\right)}{\sigma \delta\left(p_{1}+p_{2}\right)^{2}}<0, \\
& \frac{\partial \mathcal{R}_{0}}{\partial p_{2}}=\frac{-\alpha \beta\left(\mu_{n}-\sigma\right)}{\sigma \delta\left(p_{1}+p_{2}\right)^{2}}<0, \\
& \frac{\partial \mathcal{R}_{0}}{\partial \sigma}=\frac{-\alpha \beta}{\sigma \delta\left(p_{1}+p_{2}\right)}-\frac{\alpha \beta\left(\mu_{n}-\sigma\right)}{\sigma^{2} \delta\left(p_{1}+p_{2}\right)}<0 .
\end{aligned}
$$

Thus $\mathcal{R}_{0}$ is increasing with $\alpha, \beta$, and $\mu_{n}$, decreasing with $\delta, p_{1}, p_{2}$, and $\sigma$ and $\mu_{n}>\sigma$.

The following theorem defines the stability behavior of system (25) around the diseasefree equilibrium point $\mathcal{F}_{0}$. 
Theorem 3 System (25) will be locally asymptotically stable around $\mathcal{F}_{0}$ if $\mathcal{R}_{0}<1$, and it will not be stable if $\mathcal{R}_{0}>1$.

Proof Since the characteristic equation of the Jacobian matrix for (25) around $\mathcal{F}_{0}$ is

$$
(-\delta-\lambda)\left(\lambda^{2}+q_{1} \lambda+q_{2}\right)=0
$$

where $q_{1}=\sigma+p_{1}+p_{2}+\frac{\alpha \beta}{\delta}, q_{2}=\sigma\left(p_{1}+p_{2}\right)-\frac{\alpha \beta\left(\mu_{n}-\sigma\right)}{\delta}$.

The eigenvalues of Eq. (28) are $\lambda_{1}=-\delta$, and the roots of the quadratic equation

$$
\lambda^{2}+q_{1} \lambda+q_{2}=0
$$

If $q_{2}>0$, then $\mathcal{R}_{0}<1$. So

$$
\begin{aligned}
& \sigma\left(p_{1}+p_{2}\right)-\frac{\alpha \beta\left(\mu_{n}-\sigma\right)}{\delta}>0, \\
& 1>\frac{\alpha \beta\left(\mu_{n}-\sigma\right)}{\delta \sigma\left(p_{1}+p_{2}\right)} \Rightarrow \mathcal{R}_{0}<1 .
\end{aligned}
$$

Since all the parameters are positive and there is no negative term in $q_{1}$, so $q_{1}>0$. Then applying the Routh-Hurwitz criteria, we ensure that $\mathcal{F}_{0}$ is locally asymptotically stable. If $\mathcal{R}_{0}>1$, then $q_{2}<0$, and there is one positive real root for Eq. (29), thus $\mathcal{F}_{0}$ will be unstable.

\subsection{Global stability analysis of the disease-free equilibrium}

Here, global stability is calculated for DFE for system (25). The condition for model (25) which guarantees the global stability of the disease-free state is the following.

"If a model system can be written as

$$
\begin{aligned}
& \frac{d^{\gamma} U}{d \tau^{\gamma}}=H(U, W), \\
& \frac{d^{\gamma} V}{d \tau^{\gamma}}=Z(U, W), \quad Z(U, 0)=0,
\end{aligned}
$$

where $U=(S)$ and $W=(I, V)$, with $U \in \mathbb{R}_{+}^{1}$ denoting the number of uninfected individuals and $Z \in \mathbb{R}_{+}^{2}$ denoting the number of infected individuals (including the latent, the infectious, and the drug-resistant), the disease-free equilibrium is denoted by $P=\left(U^{*}, 0\right)$, where $U^{*}=\left(\frac{\alpha}{\delta}, 0\right)$.

The conditions $\left(\mathcal{H}_{1}\right)$ and $\left(\mathcal{H}_{2}\right)$ must be met to guarantee the global asymptotic stability of the disease-free equilibrium of model (25):

$$
\begin{aligned}
& \mathcal{H}_{1}: \quad \text { For } \frac{d^{\gamma} U}{d \tau^{\gamma}}=H\left(U^{*}, 0\right) \text { is globally asymptotically stable. } \\
& \mathcal{H}_{2}: \quad Z(U, W)=A W-\check{Z}(U, W), \quad \check{Z} \geq 0, \text { for }(U, W) \in \mathfrak{I}_{2},
\end{aligned}
$$

where $A=D_{W} Z\left(U^{*}, 0\right)$ is an $M$-matrix (the off-diagonal elements of $A$ are non-negative) and $\mathfrak{I}_{2}$ is the region where the model makes biological sense. If system (25) satisfies the conditions in (31), the next theorem follows. 
Theorem 4 ([7]) The fixed point $P=\left(U^{*}, 0\right)$ is globally asymptotically stable equilibrium of system (30) provided $\mathcal{R}_{0}<1$ and that the assumptions in (31) are satisfied".

Proof Let us begin by showing condition $\left(\mathcal{H}_{1}\right)$ as

$$
\begin{aligned}
& H(U, 0)=[\alpha-\delta S(\tau)], \\
& A=\left(\begin{array}{cc}
-\sigma & \frac{\beta \alpha}{\delta} \\
\mu_{n} & -\frac{\beta \alpha}{\delta}-\left(p_{1}+p_{2}\right)
\end{array}\right)
\end{aligned}
$$

and

$$
\check{Z}(U, W)=\left(\check{Z}_{11}(U, W) \quad \check{Z}_{22}(U, W)\right)=\left(\begin{array}{c}
\beta\left(\frac{\alpha}{\delta}-S\right) V \\
0
\end{array}\right) .
$$

Lemma 1 System (25) will be locally stable around $\mathcal{F}_{0}$ if $\mathcal{R}_{0}=1$.

Proof Since $\mathcal{R}_{0}=1$, then $q_{2}=0, q_{1}>0$, then the roots of Eq. (29) will be $\lambda_{2}=0, \lambda_{3}=-q_{1}$, so the system will be locally stable.

Hence we will discuss the stability of the endemic equilibrium point $\mathcal{F}^{*}$.

Definition 11 ([48]) “The discriminate $D(K)$ of a polynomial $R(\lambda)=\lambda^{3}+c_{1} \lambda^{2}+c_{2} \lambda+c_{3}$ is defined by

$$
D(K)=18 c_{1} c_{2} c_{3}+\left(c_{1} c_{2}\right)^{2}-4 c_{3}\left(c_{1}\right)^{2}-4\left(c_{2}\right)^{3}-27\left(c_{3}\right)^{2 ”} .
$$

The characteristic equation of system (25) around $\mathcal{F}^{*}$ is as follows:

$$
\lambda^{3}+c_{1} \lambda^{2}+c_{2} \lambda+c_{3}=0
$$

where

$$
\begin{aligned}
c_{1} & =\delta \mathcal{R}_{0}+\sigma+\frac{\mu_{n}\left(p_{1}+p_{2}\right)}{\left(\mu_{n}-\sigma\right)}, \\
c_{2} & =(\delta \sigma+\alpha \beta) \mathcal{R}_{0}+\frac{\alpha \beta\left(\mu_{n}-\sigma\right)}{\sigma}, \\
c_{3} & =\frac{\alpha \beta\left(\sigma-\mu_{n}\right)+\delta \sigma \mathcal{R}_{0}^{2}\left(p_{1}+p_{2}\right)}{\mathcal{R}_{0}} \\
& =\frac{\alpha \beta\left(\sigma-\mu_{n}\right)}{\mathcal{R}_{0}}+\delta \sigma \mathcal{R}_{0}\left(p_{1}+p_{2}\right) \\
& =\alpha \beta\left(\mu_{n}-\sigma\right)\left(1-\frac{1}{\mathcal{R}_{0}}\right) .
\end{aligned}
$$

Theorem 5 For $\mathcal{R}_{0}>1$ in system (25), the epidemic point $\mathcal{F}^{*}$ will be asymptotically stable if:

$$
D(K)>0, \quad c_{1} c_{2}>c_{3}, \quad \gamma \in(0,1]
$$


or

$$
D(K)<0, \quad \text { and } \quad \gamma \in\left[0, \frac{2}{3}\right)
$$

where $D(K), c_{1}, c_{2}$, and $c_{3}$ are defined in (33) and (34).

Proof For $D(K)>0, c_{1} c_{2}>c_{3}$, then $c_{1}>0, c_{3}>0$, using the Routh-Hurwitz criteria, then $|\arg (\lambda)|>\gamma \pi / 2$, and the system will be locally asymptotically stable around $\mathcal{F}^{*}$.

Since it is clear from $c_{1}>0, c_{2}>0$, and $c_{1} c_{2}>c_{3}$, the conditions for stability of the noninteger order system are satisfied [49], and so $\mathcal{F}^{*}$ is locally asymptotically stable.

\section{Numerical simulations}

Here, we calculate different scenarios for different values of fractional exponent $\gamma$ on the dynamics of dengue disease. The graphical view has been illustrated using the results of system (25). Here, three different techniques are considered using Matlab program.

Example The parameters of system (25) were chosen from the literature [45]. We can simulate any time units to see the asymptotic behavior of the given model. The units and values used in the numerical simulations of model (25) are given in Table 1.

For the disease-free equilibrium, the threshold parameter $\mathcal{R}_{0}$ has the value 0.04574 , the value below one could help control the infection. The unique equilibrium point $\mathcal{F}_{0}=(4.265,0,0)$ is asymptotically stable, this result enhances Theorem 1 . For the endemic equilibrium point, the threshold parameter $\mathcal{R}_{0}$ has the value 257.098 , the value above one and the endemic equilibrium point $\mathcal{F}^{*}=(0.5313,1.7432,10.50)$.

The effects of $\gamma$ on the dynamics of the non-integer order model (25), we conclude several numerical imitations varying the value of parameters. These simulations reveal the dynamics of the system disturbed using the value $\gamma$. Figures 5, 11, and 17 depict that for lower values of $\gamma$, the epidemic peak is wider and lower than the true equilibrium points. Figures 2-4, 6-10, 12-16, 18-19 illustrate that for lower values of $\gamma$, the epidemic peak is wider and higher for true steady states. Numerical simulations of an improved epidemic model with arbitrary order show that fractional order is related to relaxation time, i.e., the time taken to reach equilibrium. The chaotic behavior of the system when the total order of the system is less than three is sketched. A comparison between the four different values of fractional order is shown in Figures 2-19. Figures 2-4, 8-10, and 14-16 show different behaviors for $\gamma=1.00, \gamma=0.95, \gamma=0.90$, and $\gamma=0.85$. The chaotic behavior

Table 1 Parameters used and their values

\begin{tabular}{lccll}
\hline Parameter & \multicolumn{2}{l}{ Values } & Reference & Unit \\
\cline { 2 - 3 } & DFE & EE & & \\
\hline$\alpha$ & 0.56 & 0.56 & {$[45]$} & day $^{-1}$ \\
$\beta$ & 0.001 & 0.1 & {$[45]$} & day $^{-1}$ \\
$\delta$ & 0.1313 & 0.0041 & {$[45]$} & day $^{-1}$ \\
$\sigma$ & 0.5 & 0.32 & {$[45]$} & day $^{-1}$ \\
$\mu_{n}$ & 156 & 175 & {$[45]$} & day $^{-1}$ \\
$p_{1}$ & 4 & 4 & {$[45]$} & day $^{-1}$ \\
$p_{2}$ & 25 & 25 & {$[45]$} & day $^{-1}$ \\
\hline
\end{tabular}




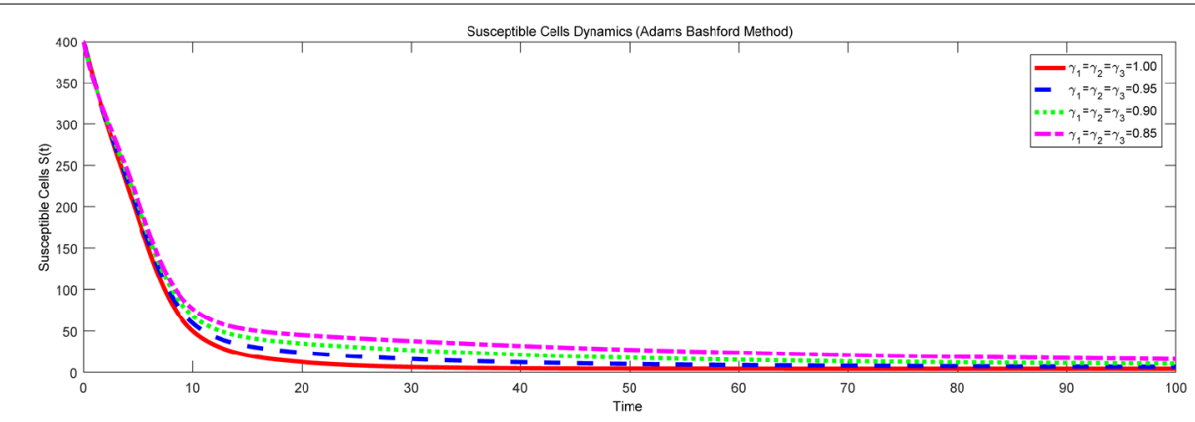

Figure 2 Dynamics of $S(t)$ with $\gamma_{i}=1$ (solid red line), $\gamma_{i}=0.95$ (dashed blue line), $\gamma_{i}=0.9$ (dotted green line) and $\gamma_{i}=0.85$ (dotted dashed magenta line), $i=1,2,3$.

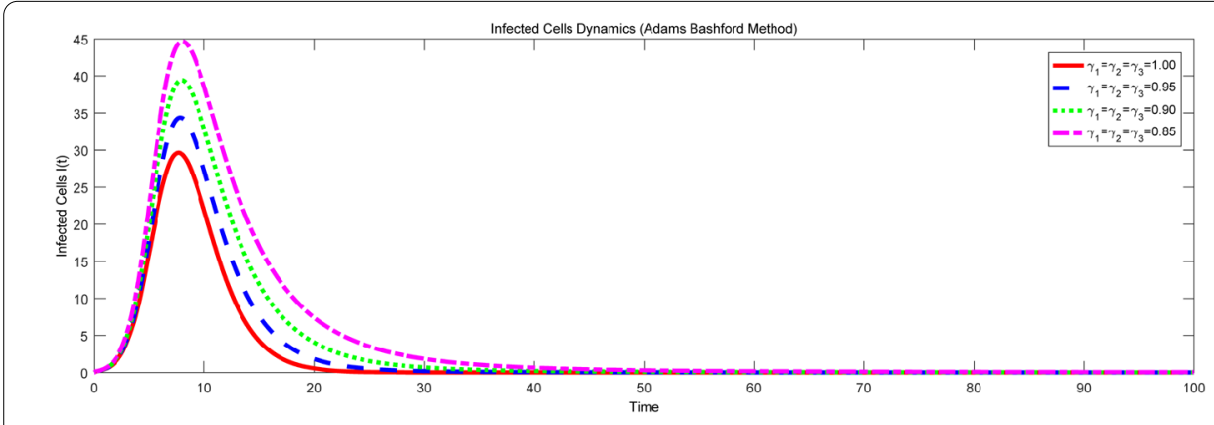

Figure 3 Dynamics of $I(t)$ with $\gamma_{i}=1$ (solid red line), $\gamma_{i}=0.95$ (dashed blue line), $\gamma_{i}=0.9$ (dotted green line) and $\gamma_{i}=0.85$ (dotted dashed magenta line), $i=1,2,3$.

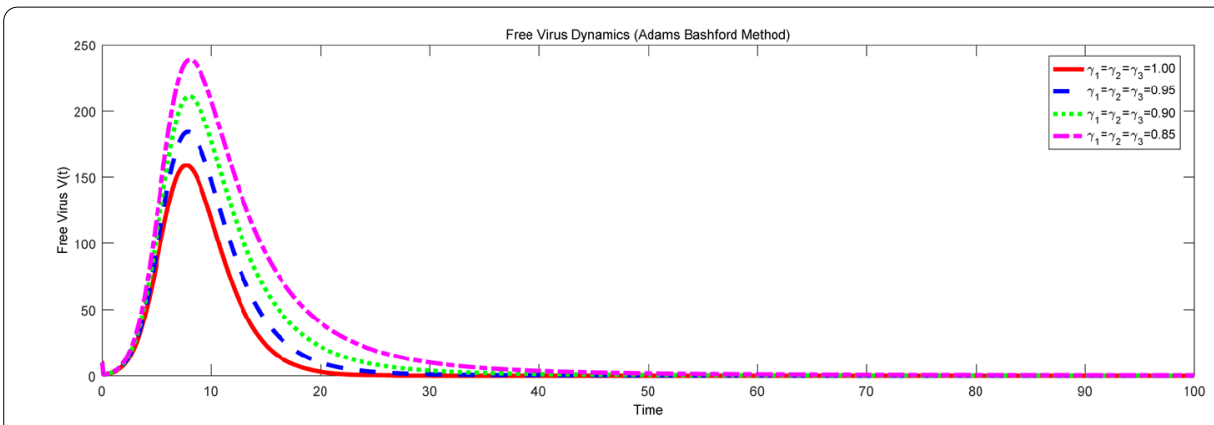

Figure 4 Dynamics of $V(t)$ with $\gamma_{i}=1$ (solid red line), $\gamma_{i}=0.95$ (dashed blue line), $\gamma_{i}=0.9$ (dotted green line) and $\gamma_{i}=0.85$ (dotted dashed magenta line), $i=1,2,3$.

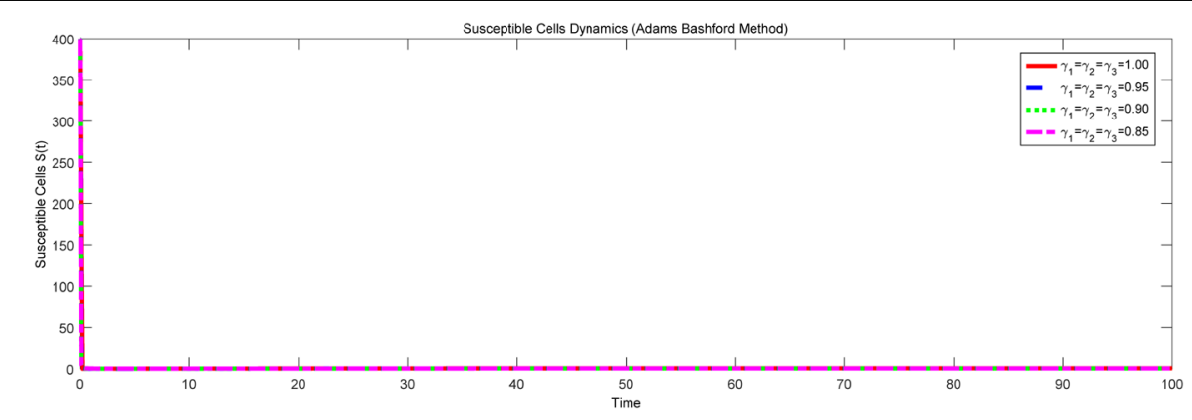

Figure 5 Dynamics of $S(t)$ with $\gamma_{i}=1$ (solid red line), $\gamma_{i}=0.95$ (dashed blue line), $\gamma_{i}=0.9$ (dotted green line) and $\gamma_{i}=0.85$ (dotted dashed magenta line), $i=1,2,3$. 


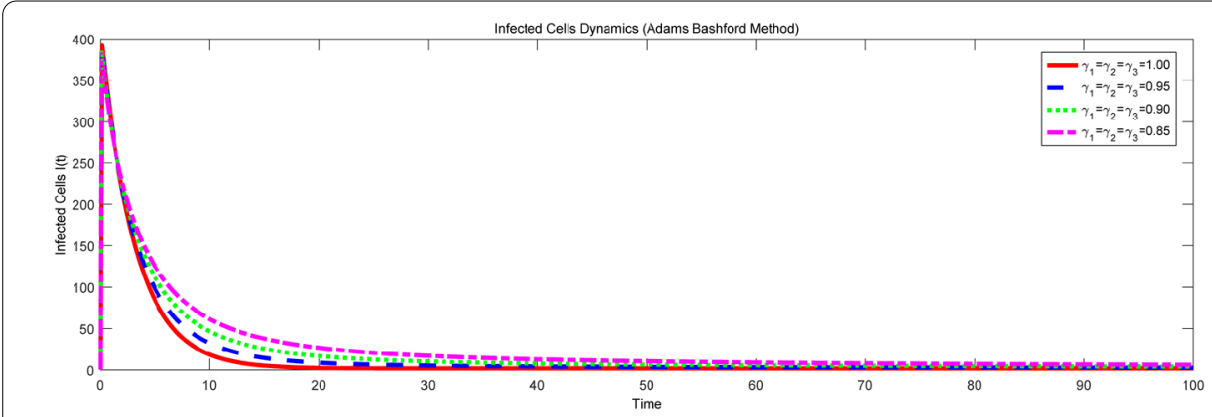

Figure 6 Dynamics of $I(t)$ with $\gamma_{i}=1$ (solid red line), $\gamma_{i}=0.95$ (dashed blue line), $\gamma_{i}=0.9$ (dotted green line) and $\gamma_{i}=0.85$ (dotted dashed magenta line), $i=1,2,3$.

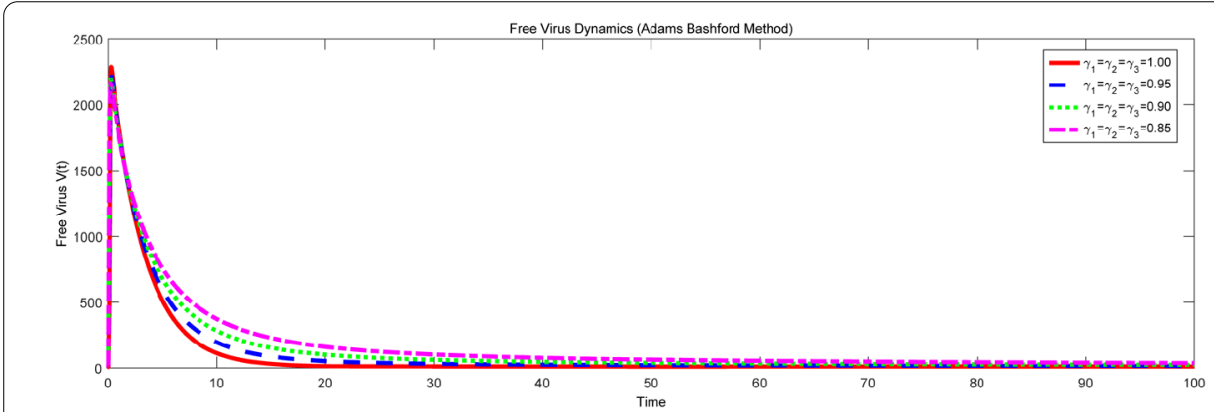

Figure 7 Dynamics of $V(t)$ with $\gamma_{i}=1$ (solid red line), $\gamma_{i}=0.95$ (dashed blue line), $\gamma_{i}=0.9$ (dotted green line) and $\gamma_{i}=0.85$ (dotted dashed magenta line), $i=1,2,3$.

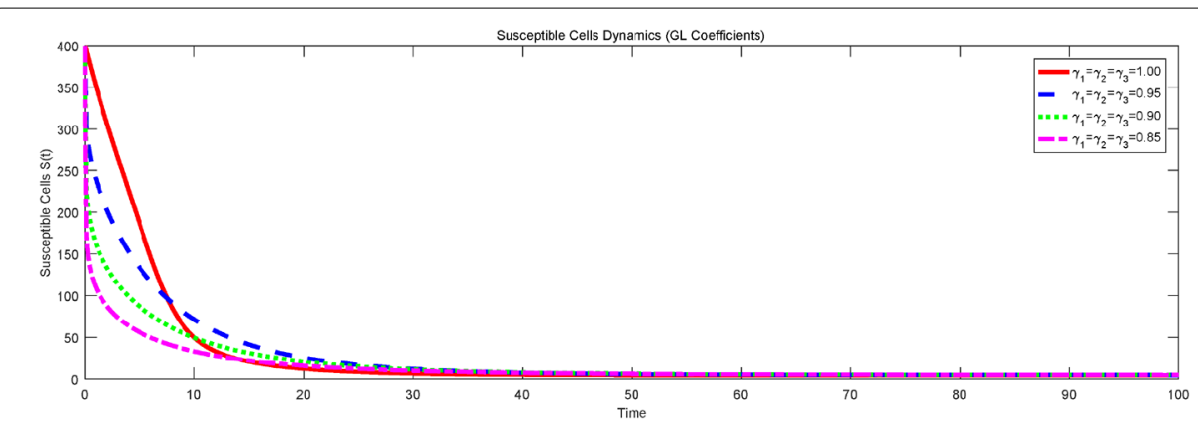

Figure 8 Dynamics of $S(t)$ with $\gamma_{i}=1$ (solid red line), $\gamma_{i}=0.95$ (dashed blue line), $\gamma_{i}=0.9$ (dotted green line) and $\gamma_{i}=0.85$ (dotted dashed magenta line), $i=1,2,3$.

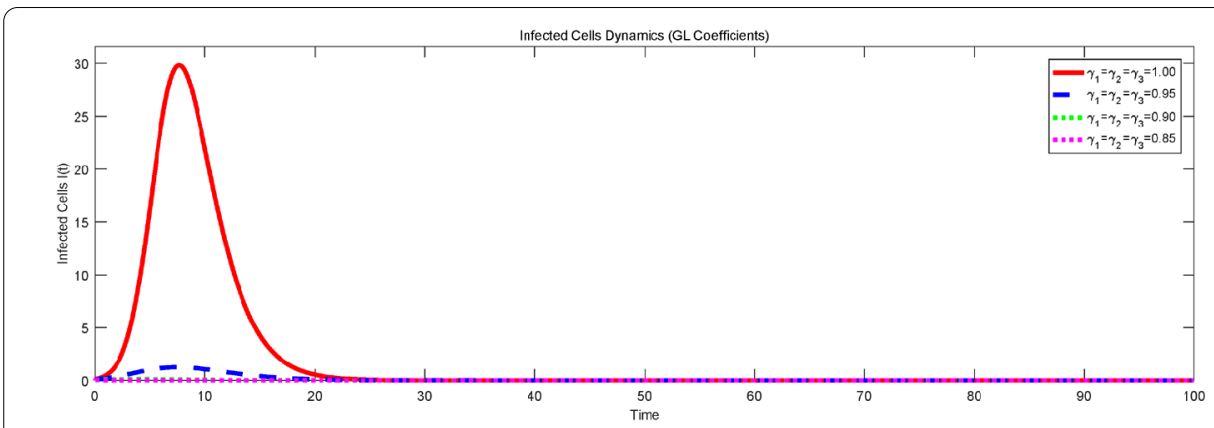

Figure 9 Dynamics of $I(t)$ with $\gamma_{i}=1$ (solid red line), $\gamma_{i}=0.95$ (dashed blue line), $\gamma_{i}=0.9$ (dotted green line) and $\gamma_{i}=0.85$ (dotted dashed magenta line), $i=1,2,3$. 


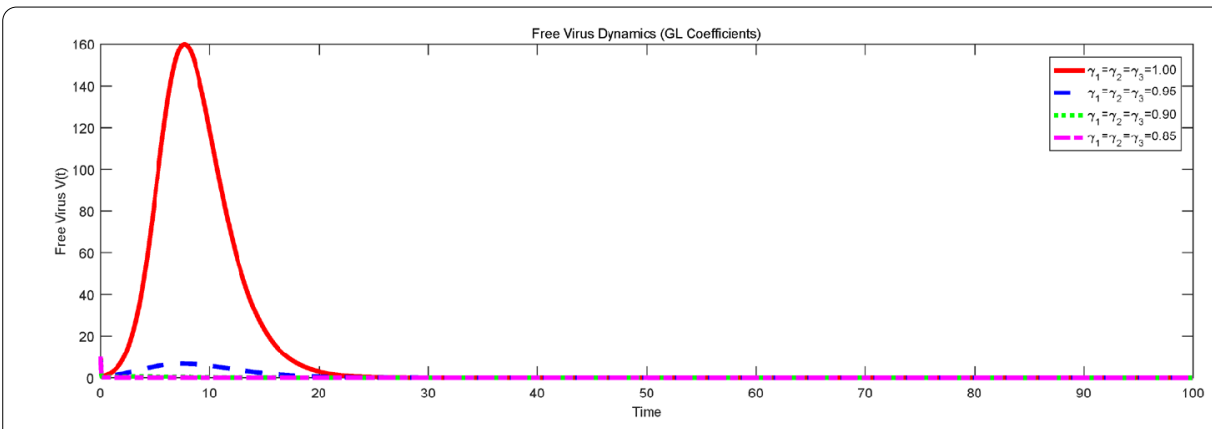

Figure 10 Dynamics of $V(t)$ with $\gamma_{i}=1$ (solid red line), $\gamma_{i}=0.95$ (dashed blue line), $\gamma_{i}=0.9$ (dotted green line) and $\gamma_{i}=0.85$ (dotted dashed magenta line), $i=1,2,3$.

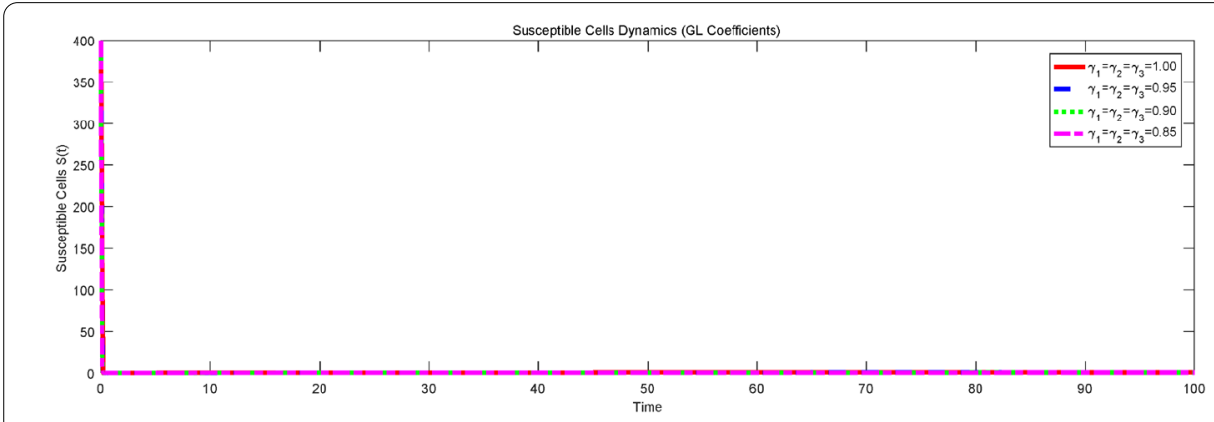

Figure 11 Dynamics of $S(t)$ with $\gamma_{i}=1$ (solid red line), $\gamma_{i}=0.95$ (dashed blue line), $\gamma_{i}=0.9$ (dotted green line) and $\gamma_{i}=0.85$ (dotted dashed magenta line), $i=1,2,3$.

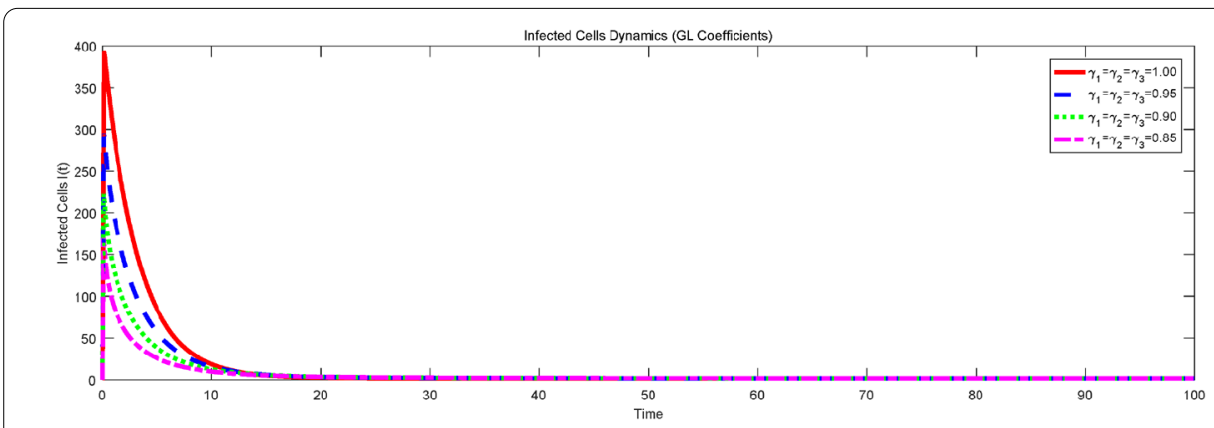

Figure 12 Dynamics of $I(t)$ with $\gamma_{i}=1$ (solid red line), $\gamma_{i}=0.95$ (dashed blue line), $\gamma_{i}=0.9$ (dotted green line) and $\gamma_{i}=0.85$ (dotted dashed magenta line), $i=1,2,3$.

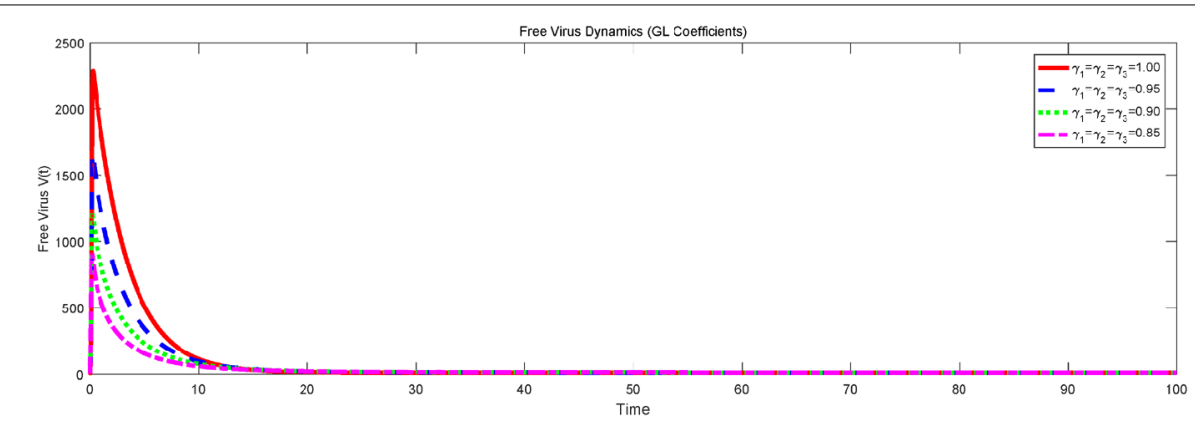

Figure 13 Dynamics of $V(t)$ with $\gamma_{i}=1$ (solid red line), $\gamma_{i}=0.95$ (dashed blue line), $\gamma_{i}=0.9$ (dotted green line) and $\gamma_{i}=0.85$ (dotted dashed magenta line), $i=1,2,3$. 


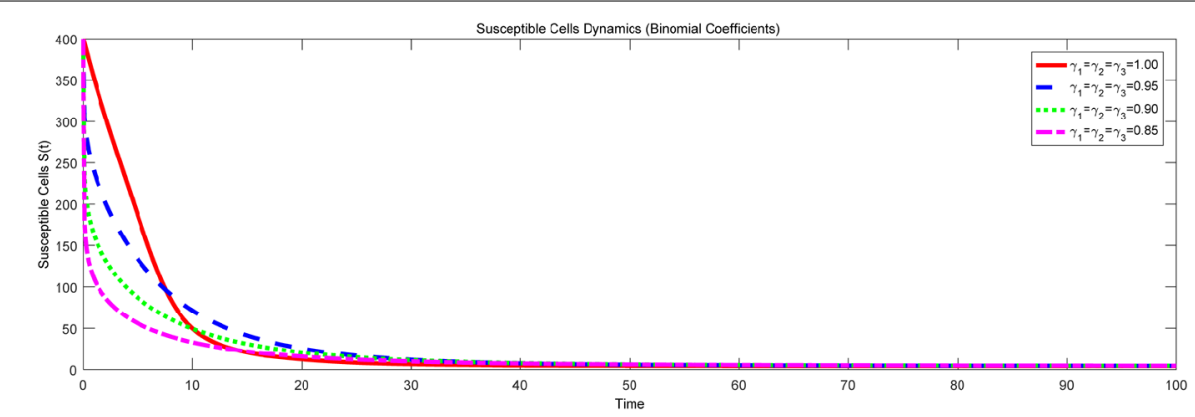

Figure 14 Dynamics of $S(t)$ with $\gamma_{i}=1$ (solid red line), $\gamma_{i}=0.95$ (dashed blue line), $\gamma_{i}=0.9$ (dotted green line) and $\gamma_{i}=0.85$ (dotted dashed magenta line), $i=1,2,3$.

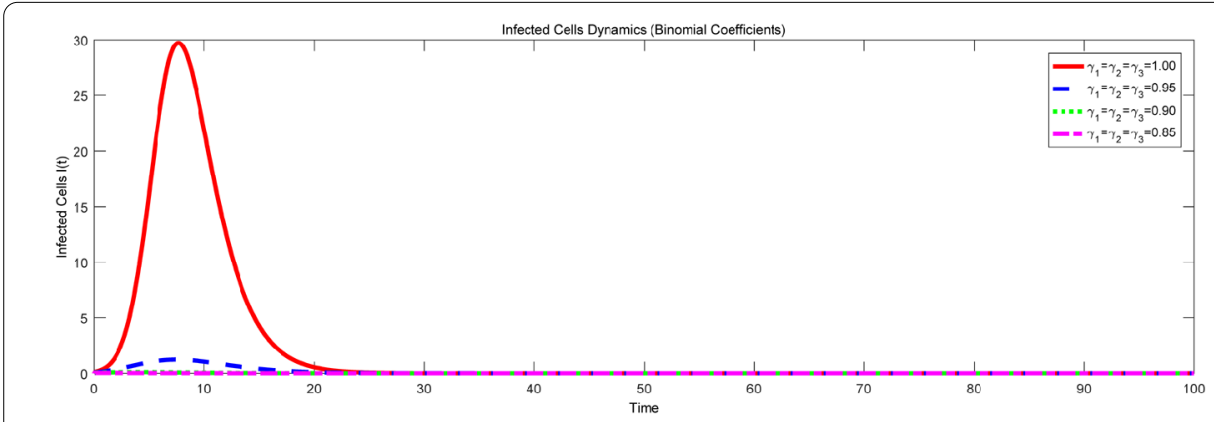

Figure 15 Dynamics of $I(t)$ with $\gamma_{i}=1$ (solid red line), $\gamma_{i}=0.95$ (dashed blue line), $\gamma_{i}=0.9$ (dotted green line) and $\gamma_{i}=0.85$ (dotted dashed magenta line), $i=1,2,3$.

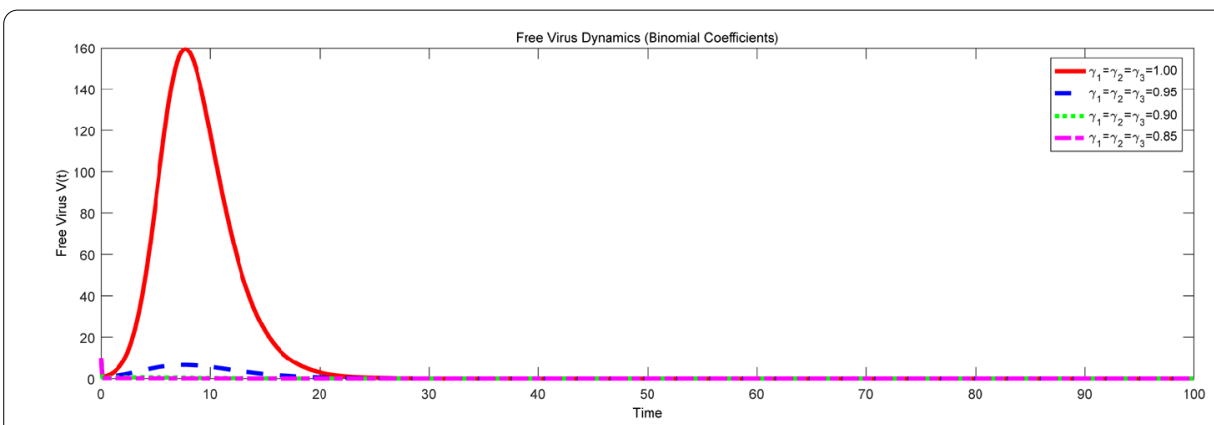

Figure 16 Dynamics of $V(t)$ with $\gamma_{i}=1$ (solid red line), $\gamma_{i}=0.95$ (dashed blue line), $\gamma_{i}=0.9$ (dotted green line) and $\gamma_{i}=0.85$ (dotted dashed magenta line), $i=1,2,3$.

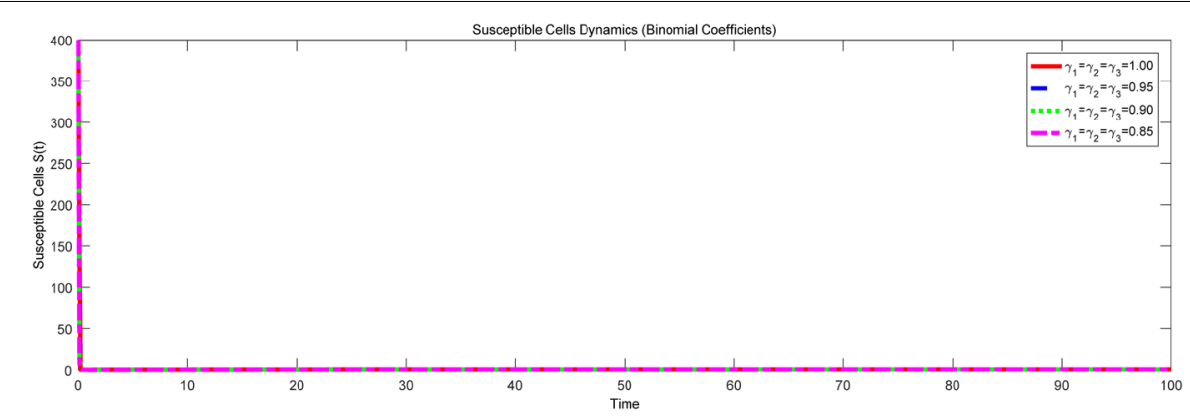

Figure 17 Dynamics of $S(t)$ with $\gamma_{i}=1$ (solid red line), $\gamma_{i}=0.95$ (dashed blue line), $\gamma_{i}=0.9$ (dotted green line) and $\gamma_{i}=0.85$ (dotted dashed magenta line), $i=1,2,3$. 


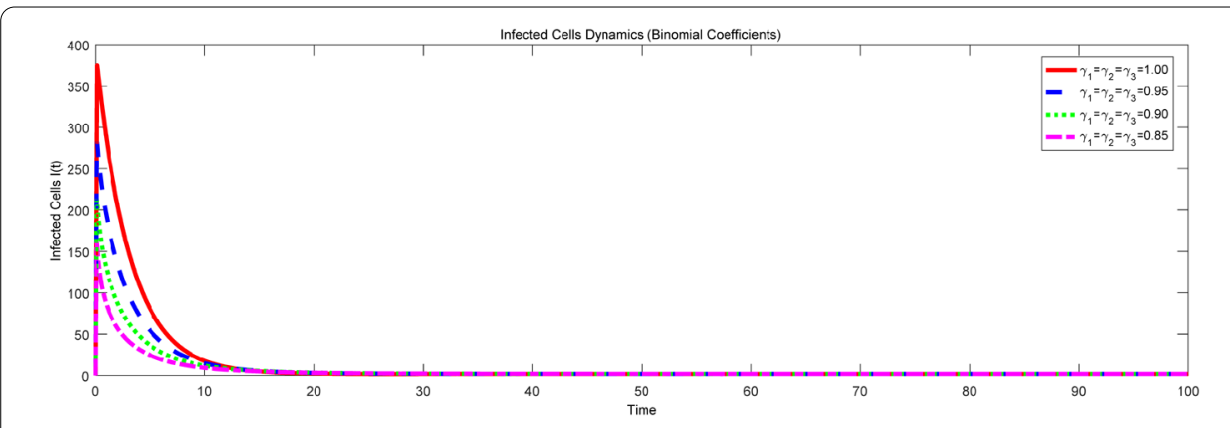

Figure 18 Dynamics of $I(t)$ with $\gamma_{i}=1$ (solid red line), $\gamma_{i}=0.95$ (dashed blue line), $\gamma_{i}=0.9$ (dotted green line) and $\gamma_{i}=0.85$ (dotted dashed magenta line), $i=1,2,3$.

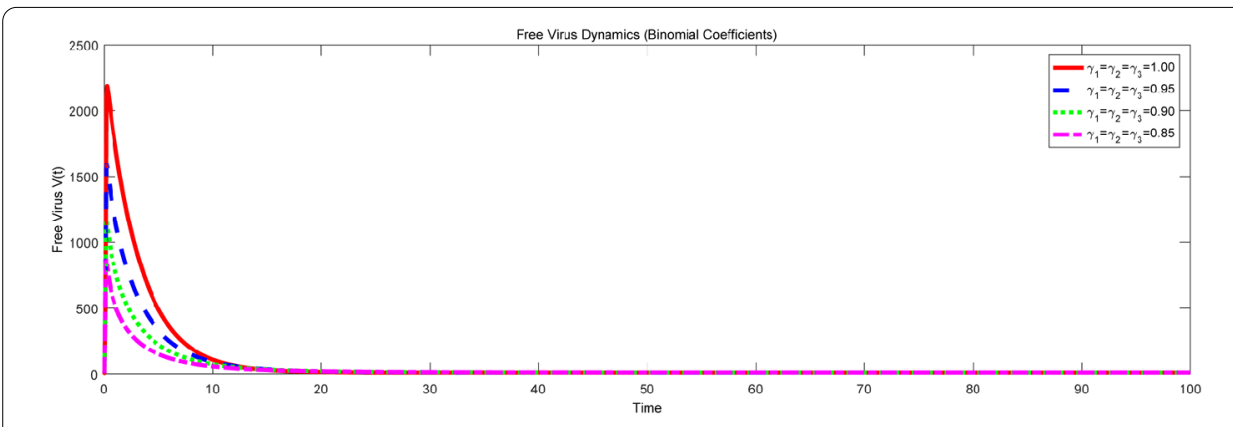

Figure 19 Dynamics of $V(t)$ with $\gamma_{i}=1$ (solid red line), $\gamma_{i}=0.95$ (dashed blue line), $\gamma_{i}=0.9$ (dotted green line) and $\gamma_{i}=0.85$ (dotted dashed magenta line), $i=1,2,3$.

of the proposed system (25) can also be seen numerically as illustrated in Tables 2 and 3. The proposed model is a fractional order model, that's why we considered three distinct orders $\gamma=0.95, \gamma=0.90, \gamma=0.85$, and the figures also illustrate different time values, e.g., time varies from 0 to 100 . And from the figures, one can check that as time varies from 0 to 100, the simulations of different orders come closer to the equilibrium point. For all four cases, the disease evolves to the disease-free and endemic equilibrium points. However, it is slower for $\gamma=0.95$, when $\gamma=0.90$, it is slower than $\gamma=0.95$, it is much slower when $\gamma=0.85$. For an epidemiological prospective, this feature is necessary for the interpreted display of a longer period in which infected ones can affect the healthy ones. Figures 2-19 illustrate that the model gradually tends towards the steady state for different $\gamma$.

\section{(i) Adams-Bashforth-Moulton algorithm}

We simulate model (25) for different values of the order of the fractional derivative, $\gamma \in[0,1]$, and biologically relevant parameters. We apply the predictor-corrector PECE method of Adams-Bashford-Moulton type [50-53].

$$
\begin{aligned}
S^{\aleph+1}= & S(0)+\frac{h^{\gamma_{1}}}{\Gamma\left(\gamma_{1}+2\right)}\left(\alpha-\beta S_{\aleph+1}^{p} V_{\aleph+1}^{p}-\delta S_{\aleph+1}^{p}\right) \\
& +\frac{h^{\gamma_{1}}}{\Gamma\left(\gamma_{1}+2\right)} \sum_{d=0}^{\aleph} a_{j, \aleph+1}\left(\alpha-\beta S_{d} V_{d}-\delta S_{d}\right)
\end{aligned}
$$


Table 2 Values of disease-free equilibrium at different time periods

\begin{tabular}{|c|c|c|c|c|}
\hline & \multicolumn{4}{|c|}{ Values of disease-free equilibrium in 100 days } \\
\hline & $\gamma_{i}=1, i=1,2,3$ & $\gamma_{i}=0.95, i=1,2,3$ & $\gamma_{i}=0.90, i=1,2,3$ & $\gamma_{i}=0.85, i=1,2,3$ \\
\hline \multicolumn{5}{|l|}{ Susceptible } \\
\hline Adams. & 4.265 & 6.597 & 10.37 & 16.02 \\
\hline Bin. coeff. & 4.265 & 4.398 & 4.491 & 4.514 \\
\hline GL coeff. & 4.265 & 4.398 & 4.491 & 4.514 \\
\hline \multicolumn{5}{|l|}{ Infected } \\
\hline Adams. & $6.181 \times 10^{-12}$ & 0.004913 & 0.01693 & 0.0454 \\
\hline Bin. coeff. & $2.034 \times 10^{-17}$ & 0.0002202 & $4.194 \times 10^{-5}$ & $1.639 \times 10^{-5}$ \\
\hline GL coeff. & $2.048 \times 10^{-17}$ & 0.000225 & $4.365 \times 10^{-5}$ & $1.742 \times 10^{-5}$ \\
\hline \multicolumn{5}{|l|}{ Free Virus } \\
\hline Adams. & $3.334 \times 10^{-11}$ & 0.02707 & 0.093 & 0.2486 \\
\hline Bin. coeff. & $1.112 \times 10^{-16}$ & 0.001204 & 0.0002293 & $8.971 \times 10^{-5}$ \\
\hline \multirow[t]{3}{*}{ GL coeff. } & $1.12 \times 10^{-16}$ & 0.00123 & 0.0002386 & $9.533 \times 10^{-5}$ \\
\hline & \multicolumn{4}{|c|}{ Values of disease-free equilibrium in 75 days } \\
\hline & $\gamma_{i}=1, i=1,2,3$ & $\gamma_{i}=0.95, i=1,2,3$ & $\gamma_{i}=0.90, i=1,2,3$ & $\gamma_{i}=0.85, i=1,2,3$ \\
\hline \multicolumn{5}{|l|}{ Susceptible } \\
\hline Adams. & 4.271 & 7.565 & 12.68 & 19.93 \\
\hline Bin. coeff. & 4.271 & 4.593 & 4.798 & 4.842 \\
\hline GL coeff. & 4.271 & 4.593 & 4.798 & 4.843 \\
\hline \multicolumn{5}{|l|}{ Infected } \\
\hline Adams. & $1.678 \times 10^{-10}$ & 0.00964 & 0.03389 & 0.0938 \\
\hline Bin. coeff. & $3.055 \times 10^{-12}$ & 0.0004264 & $7.839 \times 10^{-5}$ & $2.973 \times 10^{-5}$ \\
\hline GL coeff. & $3.067 \times 10^{-12}$ & 0.0004357 & $8.15 \times 10^{-5}$ & $3.16 \times 10^{-5}$ \\
\hline \multicolumn{5}{|l|}{ Free Virus } \\
\hline Adams. & $9.045 \times 10^{-10}$ & 0.05296 & 0.1857 & 0.5125 \\
\hline Bin. coeff. & $1.67 \times 10^{-11}$ & 0.002331 & 0.0004286 & 0.0001627 \\
\hline \multirow[t]{3}{*}{ GL coeff. } & $1.677 \times 10^{-11}$ & 0.002381 & 0.000446 & 0.0001729 \\
\hline & \multicolumn{4}{|c|}{ Values of disease-free equilibrium in 60 days } \\
\hline & $\gamma_{i}=1, i=1,2,3$ & $\gamma_{i}=0.95, i=1,2,3$ & $\gamma_{i}=0.90, i=1,2,3$ & $\gamma_{i}=0.85, i=1,2,3$ \\
\hline \multicolumn{5}{|l|}{ Susceptible } \\
\hline Adams. & 4.307 & 8.69 & 15.15 & 23.73 \\
\hline Bin. coeff. & 4.307 & 4.982 & 5.31 & 5.331 \\
\hline GL coeff. & 4.306 & 4.981 & 5.31 & 5.332 \\
\hline \multicolumn{5}{|l|}{ Infected } \\
\hline Adams. & $5.115 \times 10^{-9}$ & 0.0169 & 0.0611 & 0.1751 \\
\hline Bin. coeff. & $3.895 \times 10^{-9}$ & 0.0007376 & 0.0001308 & $4.809 \times 10^{-5}$ \\
\hline GL coeff. & $3.902 \times 10^{-9}$ & 0.0007536 & 0.0001361 & $5.112 \times 10^{-5}$ \\
\hline \multicolumn{5}{|l|}{ Free Virus } \\
\hline Adams. & $2.787 \times 10^{-8}$ & 0.09263 & 0.3342 & 0.955 \\
\hline Bin. coeff. & $2.129 \times 10^{-8}$ & 0.004032 & 0.0007151 & 0.0002632 \\
\hline GL coeff. & $2.133 \times 10^{-8}$ & 0.004118 & 0.0007442 & 0.0002797 \\
\hline
\end{tabular}

$$
\begin{aligned}
I^{\aleph+1}= & I(0)+\frac{h^{\gamma_{2}}}{\Gamma\left(\gamma_{2}+2\right)}\left(\beta S_{\aleph+1}^{p} V_{\aleph+1}^{p}-\sigma I_{\aleph+1}^{p}\right) \\
& +\frac{h^{\gamma_{2}}}{\Gamma\left(\gamma_{2}+2\right)} \sum_{d=0}^{\aleph} a_{d, \aleph+1}\left(\beta S_{d} V_{d}-\sigma I_{d}\right), \\
V^{\aleph+1}= & V(0)+\frac{h^{\gamma_{3}}}{\Gamma(\gamma+2)}\left(\mu_{n} I_{\aleph+1}^{p}-\left(p_{1}+p_{2}\right) V_{\aleph+1}^{p}-\beta S_{\aleph+1}^{p} V_{\aleph+1}^{p}\right) \\
& +\frac{h^{\gamma_{3}}}{\Gamma\left(\gamma_{3}+2\right)} \sum_{d=0}^{\aleph} a_{d, \aleph+1}\left(\mu_{n} I_{d}-\left(p_{1}+p_{2}\right) V_{d}-\beta S_{d} V_{d}\right),
\end{aligned}
$$


Table 3 Values of endemic equilibrium at different time periods

\begin{tabular}{|c|c|c|c|c|}
\hline & \multicolumn{4}{|c|}{ Values of endemic equilibrium in 100 days } \\
\hline & $\gamma_{i}=1, i=1,2,3$ & $\gamma_{i}=0.95, i=1,2,3$ & $\gamma_{i}=0.90, i=1,2,3$ & $\gamma_{i}=0.85, i=1,2,3$ \\
\hline \multicolumn{5}{|l|}{ Susceptible } \\
\hline Adams. & 0.5313 & 0.5185 & 0.5066 & 0.4969 \\
\hline Bin. coeff. & 0.5313 & 0.5266 & 0.5241 & 0.5239 \\
\hline GL coeff. & 0.5313 & 0.5264 & 0.5237 & 0.5233 \\
\hline \multicolumn{5}{|l|}{ Infected } \\
\hline Adams. & 1.743 & 2.612 & 4.006 & 6.139 \\
\hline Bin. coeff. & 1.743 & 1.757 & 1.763 & 1.761 \\
\hline GL coeff. & 1.743 & 1.758 & 1.765 & 1.762 \\
\hline \multicolumn{5}{|l|}{ Free Virus } \\
\hline Adams. & 10.5 & 15.74 & 24.14 & 37.01 \\
\hline Bin. coeff. & 10.5 & 10.58 & 10.62 & 10.61 \\
\hline \multirow[t]{3}{*}{ GL coeff. } & 10.5 & 10.59 & 10.63 & 10.62 \\
\hline & \multicolumn{4}{|c|}{ Values of endemic equilibrium in 75 days } \\
\hline & $\gamma_{i}=1, i=1,2,3$ & $\gamma_{i}=0.95, i=1,2,3$ & $\gamma_{i}=0.90, i=1,2,3$ & $\gamma_{i}=0.85, i=1,2,3$ \\
\hline \multicolumn{5}{|l|}{ Susceptible } \\
\hline Adams. & 0.5313 & 0.501 & 0.4817 & 0.4696 \\
\hline Bin. coeff. & 0.5313 & 0.5159 & 0.5068 & 0.5034 \\
\hline GL coeff. & 0.5313 & 0.5152 & 0.5055 & 0.5018 \\
\hline \multicolumn{5}{|l|}{ Infected } \\
\hline Adams. & 1.743 & 3.235 & 5.547 & 8.951 \\
\hline Bin. coeff. & 1.743 & 1.791 & 1.818 & 1.824 \\
\hline GL coeff. & 1.743 & 1.793 & 1.822 & 1.829 \\
\hline \multicolumn{5}{|l|}{ Free Virus } \\
\hline Adams. & 10.5 & 19.5 & 33.44 & 53.97 \\
\hline Bin. coeff. & 10.5 & 10.79 & 10.96 & 10.99 \\
\hline \multirow[t]{3}{*}{ GL coeff. } & 10.5 & 10.81 & 10.98 & 11.02 \\
\hline & \multicolumn{4}{|c|}{ Values of endemic equilibrium in 60 days } \\
\hline & $\gamma_{i}=1, i=1,2,3$ & $\gamma_{i}=0.95, i=1,2,3$ & $\gamma_{i}=0.90, i=1,2,3$ & $\gamma_{i}=0.85, i=1,2,3$ \\
\hline \multicolumn{5}{|l|}{ Susceptible } \\
\hline Adams. & 0.5313 & 0.5104 & 0.4945 & 0.4833 \\
\hline Bin. coeff. & 0.5313 & 0.5222 & 0.5168 & 0.5153 \\
\hline GL coeff. & 0.5313 & 0.5217 & 0.516 & 0.5142 \\
\hline \multicolumn{5}{|l|}{ Infected } \\
\hline Adams. & 1.743 & 2.961 & 4.761 & 7.528 \\
\hline Bin. coeff. & 1.743 & 1.771 & 1.786 & 1.786 \\
\hline GL coeff. & 1.743 & 1.773 & 1.788 & 1.790 \\
\hline \multicolumn{5}{|l|}{ Free Virus } \\
\hline Adams. & 10.5 & 17.57 & 28.7 & 45.39 \\
\hline Bin. coeff. & 10.5 & 10.67 & 10.76 & 10.76 \\
\hline GL coeff. & 10.5 & 10.68 & 10.78 & 10.78 \\
\hline
\end{tabular}

where

$$
\begin{aligned}
& S_{\aleph+1}^{p}=S(0)+\frac{1}{\Gamma\left(\gamma_{1}\right)} \sum_{d=0}^{\aleph} b_{d, \aleph+1}\left(\alpha-\beta S_{d} V_{d}-\delta S_{d}\right), \\
& I_{\aleph+1}^{p}=I(0)+\frac{1}{\Gamma\left(\gamma_{2}\right)} \sum_{d=0}^{\aleph} b_{d, \aleph+1}\left(\beta S_{d} V_{d}-\sigma I_{d}\right), \\
& V_{\aleph+1}^{p}=V(0)+\frac{1}{\Gamma\left(\gamma_{3}\right)} \sum_{d=0}^{\aleph} b_{d, \aleph+1}\left(\mu_{n} I_{d}-\left(p_{1}+p_{2}\right) V_{d}-\beta S_{d} V_{d}\right),
\end{aligned}
$$


with

$$
a_{d, \aleph+1}= \begin{cases}\aleph_{\gamma_{i}+1}-\left(\aleph-\gamma_{i}\right)(\aleph+1)^{\gamma_{i},} & d=0, \\ (\aleph-d+2)^{\gamma_{i}+1}+(\aleph-d)^{\gamma_{i}+1}-2(\aleph-d+1)^{\gamma_{i}+1}, & 1 \leq d \leq \aleph, \\ 1, & d=\aleph+1\end{cases}
$$

and

$$
b_{d, \aleph+1}=\frac{h^{\gamma_{i}}}{\gamma_{i}}\left((\aleph-d+1)^{\gamma_{i}}-(\aleph-d)^{\gamma_{i}}\right), \quad 0 \leq d \leq \aleph
$$

with $i=1,2,3$.

Disease-free equilibrium (Figures 2-4).

Endemic equilibrium (Figures 5-7).

(ii) GL algorithm (GL coefficients)

Here we simulate model (25) for different values of the order of the fractional derivative, $\gamma \in[0,1]$, and biologically relevant parameters. We apply the Grunwald-Letnikov method by using the GL-coefficient $[37,54,55]$.

$$
\begin{aligned}
& \sum_{j=0}^{\aleph+1} P_{j}^{\gamma_{1}} S^{\aleph+1-j}=\alpha-\beta S^{\aleph+1} V^{\aleph}-\delta S^{\aleph+1}, \\
& \sum_{j=0}^{\aleph+1} P_{j}^{\gamma_{2}} I^{\aleph+1-j}=\beta S^{\aleph+1} V^{\aleph}-\sigma I^{\aleph+1}, \\
& \sum_{j=0}^{\aleph+1} P_{j}^{\gamma_{3}} V^{\aleph+1-j}=\mu_{n} I^{\aleph+1}-\beta S^{\aleph+1} V^{\aleph+1}-\left(p_{1}+p_{2}\right) V^{\aleph+1}, \\
& (37) \Rightarrow S^{\aleph+1}=\frac{\alpha-\sum_{j=1}^{\aleph+1} P_{j}^{\gamma_{1}} S^{\aleph+1-j}}{P_{0}^{\chi 1}+\beta V^{n}+\delta}, \\
& (38) \Rightarrow I^{\aleph+1}=\frac{\beta S^{\aleph+1} V^{\aleph}-\sum_{j=1}^{\aleph+1} P_{j}^{\gamma_{2}} I^{\aleph+1-j}}{P_{0}^{\chi 2}+\sigma}, \\
& (39) \Rightarrow V^{\aleph+1}=\frac{\mu_{n} I^{\aleph+1}-\sum_{j=1}^{\aleph+1} P_{j}^{\gamma_{3}}\left(Y_{2}\right)^{\aleph+1-j}}{P_{0}^{\chi 3}+\beta S^{\aleph+1}+\left(p_{1}+p_{2}\right)}
\end{aligned}
$$

with $P_{0}^{\gamma_{1}}=\left(\frac{(\delta) h_{-1}}{\delta}\right)^{-\gamma_{1}}, P_{0}^{\gamma_{2}}=\left(\frac{e^{(\sigma) h_{-1}}}{\sigma}\right)^{-\gamma_{2}}, P_{0}^{\gamma_{3}}=\left(\frac{e^{\left(p_{1}+p_{2}\right) h_{-1}}}{p_{1}+p_{2}}\right)^{-\gamma_{3}}$ and $P_{i}^{\gamma_{m}}=\left(1-\frac{1+\gamma_{m}}{i}\right) P_{i-1}^{\gamma_{m}}, m=$ $1,2,3$.

Disease-free equilibrium (Figures 8-10).

Endemic equilibrium (Figures 11-13).

(iii) Grunwald-Letnikov algorithm (binomial coefficients)

Here we simulate model (25) for different values of the order of the fractional derivative, $\gamma \in[0,1]$, and biologically relevant parameters. We apply the Grunwald-Letnikov method 
by using binomial coefficients $[48,56-58]$.

$$
\begin{aligned}
& S\left(t_{\aleph}\right)=\left\{\alpha-\beta S\left(t_{\aleph-1}\right) V\left(t_{\aleph-1}\right)-\delta S\left(t_{\aleph-1}\right)\right\} h^{\gamma_{1}}-\sum_{j=1}^{\aleph} P_{j}^{\left(\gamma_{1}\right)} S\left(t_{\aleph-j}\right), \\
& I\left(t_{\aleph}\right)=\left\{\beta S\left(t_{\aleph}\right) V\left(t_{\aleph-1}\right)-\sigma I\left(t_{\aleph-1}\right)\right\} h^{\gamma_{2}}-\sum_{j=1}^{\aleph} P_{j}^{\left(\gamma_{2}\right)} I\left(t_{\aleph-j}\right), \\
& V\left(t_{\aleph}\right)=\left\{\mu_{n} I\left(t_{\aleph}\right)-\left(p_{1}+p_{2}\right) V\left(t_{\aleph-1}\right)-\beta S\left(t_{\aleph}\right) V\left(t_{\aleph-1}\right)\right\} h^{\gamma_{3}}-\sum_{j=1}^{\aleph} P_{j}^{\left(\gamma_{3}\right)} V\left(t_{\aleph-j}\right),
\end{aligned}
$$

where $P_{0}^{\left(\gamma_{i}\right)}=1, P_{j}^{\left(\gamma_{i}\right)}=\left(1-j^{-1}\left(1+\gamma_{i}\right)\right) P_{j-1}^{\left(\gamma_{i}\right)}, i=1,2,3$.

Disease-free equilibrium (Figures 14-16).

Endemic equilibrium (Figures 17-19).

\section{Conclusion}

A nonlinear mathematical dengue model with fractional order $\gamma_{i}, i=1,2,3$, is formulated. The stability of both DFE and EE points is discussed. Sufficient conditions for local stability of the DFE point $\mathcal{F}_{0}$ are given in terms of the basic reproduction number $\mathcal{R}_{0}$ of the model, where it is asymptotically stable if $\mathcal{R}_{0}<1$. The positive infected equilibrium $\mathcal{F}^{*}$ exists when $\mathcal{R}_{0}>1$, and sufficient conditions that guarantee the asymptotic stability of this point are calculated. Besides this sensitivity analysis of the parameters involved, the threshold parameter $\left(\mathcal{R}_{0}\right)$ is also discussed. Three fractional order techniques are used to check the best performance of the model. When simulating the model with all three algorithms, we have observed that all methods are converging to the disease-free and endemic equilibrium points through different paths and for different values of $\gamma_{i}, i=1,2,3$. The values are very close to each other in all three techniques as given in Tables 2 and 3 . However, the time consumed (Core i5 laptop) by Grunwald-Letnikov (binomial coefficient) is $3125.817 \mathrm{sec} \approx 52 \mathrm{~min}$, by Grunwald-Letnikov (GL coefficient) is $2483.031 \mathrm{sec} \approx 41 \mathrm{~min}$, and by the Adams-Bashforth-Moulton algorithm is $67326.743 \mathrm{sec} \approx 18.7 \mathrm{hrs}$, which indicates that the computational cost for Grunwald-Letnikov (GL coefficients) is cheaper than that for the other two. Dengue is one of the most rapidly spreading mosquito-borne viral diseases in the world, and it inflicts significant health, economics, and social burdens on populations. The main purpose of analyzing the dengue model with these techniques is that it helps the researchers and policy makers in targeting, prevention, and treatment resources for maximum effectiveness. Numerical simulations with different order show that the system decays to the equilibrium condition, e.g., power of $t^{-\gamma}$. The result provides an important insight into the use of fractional order to model the dengue internal disease. The order of the fractional derivative $\gamma$ may be associated with differences in individuals' immune system, age, treatment compliance, treatment toxicities and other co-morbidities, amongst others. The fractional order may provide more 'freedom' to adjust the model to real data of specific patients. That is to say, the fractional order index contributes positively to better fit the patient data.

In the future, optimal control strategies can be incorporated into the proposed model for the control of virus inside the body. 


\section{Acknowledgements}

We would like to thank the referees for their valuable comments.

\section{Competing interests}

The authors declare that they have no competing interests.

\section{Authors' contributions}

The authors have achieved equal contributions. All authors read and approved the manuscript.

\section{Author details}

'Department of Mathematics, University of Engineering and Technology, Lahore, Pakistan. ${ }^{2}$ Faculty of Information Technology, University of Central Punjab, Lahore, Pakistan. ${ }^{3}$ Department of Mathematics, University of Engineering and Technology, KSK Campus, Lahore, Pakistan.

\section{Publisher's Note}

Springer Nature remains neutral with regard to jurisdictional claims in published maps and institutional affiliations.

\section{Received: 28 September 2017 Accepted: 3 January 2018 Published online: 18 January 2018}

\section{References}

1. Guzman, MG, Kouri, G: Dengue: an update. Lancet Infect. Dis. 2(1), 33-42 (2002)

2. Malaviage, GN, Fernando, S, Fernando, DJ, Seneviratne, SL: Dengue viral infections. Postgrad. Med. J. 80, 588-601 (2004)

3. Baleanu, D, Golmankhaneh, AK, Golmankhaneh, AK, Nigmatullin, RR: Newtonian law with memory. Nonlinear Dyn. $60,81-86(2010)$

4. Baleanu, D, Mustafa, OG: On the global existence of solutions to a class of fractional differential equations. Comput. Math. Appl. 59, 1835-1841 (2010)

5. Agila, A, Baleanu, D, Eid, R, Irfanoglu, B: Applications of the extended fractional Euler-Lagrange equations model to freely oscillating dynamical systems. Rom. J. Phys. 61(3-4), 350-359 (2016)

6. Du, M, Wang, Z, Hu, H: Measuring memory with the order of fractional derivative. Sci. Rep. 3, 3431-3434 (2013)

7. Pinto, CMA, Carvalho, ARM: The HIV/TB coinfection severity in the presence of TB multi-drug resistant strains. Ecol. Complex. 23, 1-20 (2017)

8. Skwara, U, Martins, J, Ghaffari, P, Aquiar, M, Boto, J, Stollenwerk, N: Fractional calculus and superdiffusion in epidemiology: shift of critical thresholds. In: Proceedings of the 12th International Conference on Computational and Mathematical Methods in Science and Engineering, La Manga (2012)

9. West, BJ, Grigolini, P, Metzler, R, Nonnenmacher, TF: Fractional diffusion and Lévy stable processes. Phys. Rev. E 55(1), 99-106 (1997)

10. Metzler, R, Klafter, J: The random walk's guide to anomalous diffusion: a fractional dynamics approach. Phys. Rep. 339 1-77 (2000)

11. Golmankhaneh, AK, Arefi, R, Baleanu, D: Synchronization in a nonidentical fractional order of a proposed modified system. J. Vib. Control 21(6), 1154-1161 (2015)

12. Ozalp, N, Demirci, E: A fractional order SEIR model with vertical transmission. Math. Comput. Model. 54, 1-6 (2011)

13. Liu, Z, Lu, P: Stability analysis for HIV infection of CD4+ T-cells by a fractional differential time-delay model with cure rate. Adv. Differ. Equ. 2014, Article ID 298 (2014)

14. Doungmo Goufo, EF, Maritz, R, Munganga, J: Some properties of the Kermack-McKenderick epidemic model with fractional derivative and nonlinear incidence. Adv. Differ. Equ. 2014, Article ID 278 (2014)

15. Doungmo Goufo, EF: Stability and convergence analysis of a variable order replicator-mutator process in a moving medium. J. Theor. Biol. 403, 178-187 (2016)

16. Al-Sulami, H, El-Shahed, M, Nieto, JJ, Shammakh, W: On fractional order dengue epidemic model. Math. Probl. Eng. (2014). https://doi.org/10.1155/2014/456537

17. Diethelm, K, Freed, AD: The fractional PECE subroutine for the numerical solution of differential equations of fractional order. http://www.mpg.de/billing/hbp98/diethelm.pdf (2002)

18. Purohit, SD, Kalla, SL: On fractional partial differential equations related to quantum mechanics. J. Phys. A, Math. Theor. 44(4), 045202 (2011)

19. Purohit, SD: Solutions of fractional partial differential equations of quantum mechanics. Adv. Appl. Math. Mech. 5(5), 639-651 (2013)

20. Chouhan, A, Purohit, SD, Saraswat, S: An alternate method for solving generalized differential equations of fractional order. Kragujev. J. Math. 37(2), 299-306 (2013)

21. Nisar, KS, Purohit, SD, Mondal, SR: Generalized fractional kinetic equations involving generalized Struv function of the first kind. J. King Saud Univ., Sci. 28(2), 167-171 (2016)

22. Zafar, Z, Rehan, K, Mushtaq, M: Fractional-order scheme for bovine babesiosis disease and tick populations. Adv Differ. Equ. 2017, Article ID 86 (2017)

23. Zafar, Z, Rehan, K, Mushtaq, M, Rafiq, M: Numerical treatment for nonlinear brusselator chemical model. J. Differ. Equ. Appl. 23(3), 521-538 (2017)

24. Zafar, Z, Rehan, K, Mushtaq, M, Rafiq, M: Numerical modeling for nonlinear biochemical reaction networks. Iran. J. Math. Chem. 8(4), 413-423 (2017)

25. Zafar, Z, Ahmad, MO, Pervaiz, A, Rafiq, M: Fourth order compact method for one dimensional inhomogeneous telegraph equations with $O\left(h^{4}, k^{3}\right)$. Pak. J. Eng. Appl. Sci. 14, 96-101 (2014)

26. Zafar, Z, Rehan, K, Mushtaq, M, Rafiq, M: HIV/AIDS epidemic fractional-order model. J. Differ. Equ. Appl. 23(7), 1298-1315 (2017) 
27. Baleanu, D, Diethelm, K, Scalas, E, Trujillo, J: Fractional Calculus: Models and Numerical Methods, vol. 3. World Scientific, Singapore (2012)

28. Coronel-Escamilla, A, Torres, F, Gomez-Aguilar, JF, Escobar-Jimenez, RF, Guerrero, GV: On the trajectory tracking control for an SCARA robot manipulator in a fractional model driven by induction motors with PSO tuning. Multibody Syst. Dyn. (2017). https://doi.org/10.1007/s11044-017-9586-3

29. Coronel-Escamilla, A, Gomez-Aguilar, JF, Lopez-Lopez, MG, Alvarado-Martinez, VM: Triple pendulum model involving fractional derivatives with different kernels. Chaos Solitons Fractals 91, 248-261 (2016)

30. Gomez-Aguilar, JF, Baleanu, D: Schrodinger equation involving fractional operators with non-singular kernel. J. Electromagn. Waves Appl. (2017). https://doi.org/10.1080/09205071.2017.1312556

31. Gomez-Aguilar, JF, Yepez-Martinez, H, Torres-Jimenez, J, Cordova-Fraga, T, Escobar-Jimenez, RF, Olivares-Peregrino, WH: Homotopy perturbation transform method for nonlinear differential equations involving to fractional operator with exponential kernel. Adv. Differ. Equ. 2017, Article ID 68 (2017)

32. Morales-Delgado, VF, Taneco-Hernandez, MA, Gomez-Aguilar, JF: On the solutions of fractional order of evolution equations. Eur. Phys. J. Plus 132(47), 1-14 (2017)

33. Gomez-Aguilar, JF, Yepez-Martinez, H, Escobar-Jimenez, RF, Astorga-Zaragoza, CM, Reyes-Reyes, J: Analytical and numerical solutions of electrical circuits described by fractional derivatives. Appl. Math. Model. (2016). https://doi.org/10.1016/j.apm.2016.05.041

34. Carvalho, ARM, Pinto, CMA: Within-host and synaptic transmissions: contributions to the spread of HIV infection. Math. Methods Appl. Sci. (2016). https://doi.org/10.1002/mma.4047

35. Pinto, CMA, Carvalho, ARM: The role of synaptic transmission in a HIV model with memory. Appl. Math. Comput. 292, 76-95 (2017)

36. Shim, E: A note on epidemic models with infective immigrants and vaccination. Math. Biosci. Eng. 3, 557-566 (2006)

37. Zibaei, S, Namjoo, M: A nonstandard finite difference scheme for solving fractional-order model of HIV-1 infection of CD4+ T-cells. Iran. J. Math. Chem. 6, 169-184 (2015)

38. Miller, KS, Ross, B: An Introduction to the Fractional Calculus and Fractional Differential Equations. Wiley, New York (1993)

39. Caputo, M, Fabrizio, M: A new definition of fractional derivative without singular kernel. Prog. Fract. Differ. Appl. 1(2), 73-85 (2015)

40. Losada, J, Nieto, JJ: Properties of a new fractional derivative without singular kernel. Prog. Fract. Differ. Appl. 1(2), 87-92 (2015)

41. Goufo, EFD: Application of the Caputo-Fabrizio fractional derivative without singular kernel to Korteweg-deVries-Burgers equation. Math. Model. Anal. 21(2), 188-198 (2016)

42. Goufo, EFD: Chaotic processes using the two-parameter derivative with non-singular and non-local kernel: basic theory and application. Chaos 26, 084305 (2016). https://doi.org/10.1063/1.4958921

43. Khalil, R, Al-Horani, M, Yousef, A, Sababheh, M: A new definition of the fractional derivative. J. Comput. Appl. Math. 264, 65-70 (2014)

44. Kurane, I, Takasaki, T: Dengue fever and dengue haemorrhagic fever: challenges of controlling an enemy still at large. Rev. Med. Virol. 11, 301-311 (2001)

45. Nurani, N, Soewono, E, Sidarto, KA: A mathematical model of dengue internal transmission process. J. Indones. Math. Soc. 13(1), 123-132 (2007)

46. Nowak, MA, May, RM: Virus Dynamics: Mathematical Principles of Immunology and Virology. Oxford University Press, New York (2000)

47. Podlubny, I: Fractional Differential Equations. Academic Press, San Diego (1999)

48. Ahmed, E, El-Sayed, AMA, El-Saka, HAA: On some Routh-Hurwitz conditions for fractional order differential equations and their applications in Lorenz, Rossler, Chua and Chen systems. Phys. Lett. A 358, 1-4 (2006)

49. Radwan, AG, Moaddy, K, Momani, S: Stability and non-standard finite difference method of the generalized Chua's circuit. Comput. Math. Appl. 62, 961-970 (2011)

50. Li, C, Tao, C: On the fractional Adams method. Comput. Math. Appl. 58, 1573-1588 (2009)

51. Diethelm, K: An algorithm for the numerical solution of differential equations of fractional order. Electron. Trans. Numer. Anal. 5, 1-6 (1997)

52. Diethelm, K, Ford, NJ: Analysis of fractional differential equations. J. Math. Anal. Appl. 265, 229-248 (2002)

53. Diethelm, K, Ford, NJ, Freed, AD: A predictor-corrector approach for the numerical solution of fractional differential equations. Nonlinear Dyn. 29, 3-22 (2002)

54. Wang, X, Yang, J, Li, X: Dynamics of a heroin epidemic model with very population. Appl. Math. 2, $732-738$ (2011)

55. Mickens, RE: Numerical integration of population models satisfying conservation laws: NSFD methods. J. Biol. Dyn. 1(4), 1751-1766 (2007)

56. Vinagre, BM, Chen, YQ, Petras, I: Two direct tustin discretization methods for fractional order differentiator/integrator. J. Franklin Inst. 340, 349-362 (2003)

57. Dorcak, L: Numerical models for the simulation of the fractional order control systems, in UEF-04-94, The Academy of Sciences, Institute of Experimental Physics, Kosice (1994)

58. Pinto, CMA, Carvalho, ARM: A latency fractional order model for HIV dynamics. J. Comput. Appl. Math. (2017). https://doi.org/10.1016/j.cam.2016.05.019 